ESAIM: PROCEEDINGS, December 2012, Vol. 38, p. 275-297

F. Coquel, M. Gutnic, P. Helluy, F. Lagoutière, C. Rohde, N. Seguin, Editors

\title{
FINITE VOLUME SCHEMES FOR VLASOV
}

\author{
Nicolas Crouseilles ${ }^{1}$, Pierre Glanc ${ }^{2}$, Michel Mehrenberger ${ }^{2}$ and Christophe \\ STEINER $^{2}$
}

\begin{abstract}
We present finite volume schemes for the numerical approximation of the one-dimensional Vlasov-Poisson equation (FOV CEMRACS 2011 project). Stability analysis is performed for the linear advection and links with semi-Lagrangian schemes are made. Finally, numerical results enable to compare the different methods using classical plasma test cases.

Résumé. Des schémas de type volumes finis sont étudiés ici pour l'approximation de l'équation de Vlasov-Poisson (projet FOV, CEMRACS 2011). Une analyse de stabilité est effectuée dans le cas de l'advection linéaire et plusieurs liens sont faits entre les méthodes volumes finis et semi-Lagrangiennes. Enfin, les méthodes sont comparées sur des cas tests académiques de la physique des plasmas.
\end{abstract}

\section{INTRODUCTION}

The description of a plasma can be performed at the kinetic level by the Vlasov-Poisson system. In a reduced problem, its solution $f(t, x, v)$ depends on time $t \geq 0$, on space $x \in[0, L]$ and on velocity $v \in \mathbb{R}$ and satisfies the following equation

$$
\partial_{t} f+v \partial_{x} f+E \partial_{v} f=0, \quad \partial_{x} E=\int_{\mathbb{R}} f d v-1,
$$

with $E(t, x)$ the self-consistent electric field which verifies $\int_{0}^{L} E(t, x) d x=1$. An initial condition $f_{0}(x, v)$ is added to this system ; moreover periodic boundary conditions in space and vanishing in velocity are considered.

Due to the nonlinearity of the problem, it is difficult to find analytical solution to (1) so that numerical methods have been proposed to solve it. Historically, the first one has been particles methods (see [3]) which consists in advancing in time macro-particles through the equations of motion. Despite the inherent numerical noise which prevents precise description of low density regions, there are still a huge use of these methods used due to their low computational cost. On the other side, Eulerian methods which have been developed more recently use a grid of phase space $(x, v)$. Among them, we can cite finite volumes methods and semi-Lagrangian methods. These methods are often used in a splitting framework ; typically, to solve (1), the strategy decomposes the multi-dimensional problem in several $1 \mathrm{D}$ problems. We refer to $[1,6,9,13,16,17,21]$ for previous works on the subject. Even if the Vlasov-Poisson context enables a licit splitting operator, in some situations however this procedure is not suitable (see $[7,19]$ ) and can lead to numerical instabilities. The main goal of this work is to look for unsplit versions of finite volumes schemes. Such schemes have already been developed in [15] and more recently in [4].

\footnotetext{
${ }^{1}$ INRIA-Rennes Bretagne Atlantique, projet IPSO \& IRMAR, Université de Rennes 1, 263 avenue du général Leclerc, 35042 Rennes.

2 INRIA-Nancy Grand-Est, projet CALVI \& IRMA, Université de Strasbourg, 7, rue René Descartes, 67084 Strasbourg.
}

(c) EDP Sciences, SMAI 2012 
We will detail here two types of strategy. The first one follows [4] and leads to a system of ODE's, with upwind or central spatial approximations. The second strategy consists in approximating the flux with Gauss points which are evaluated by solving backward in time the characteristics ; this enables to avoid the transport of $2 \mathrm{D}$ volumes, which leads to mesh intersection computations (see [20]). Other strategies in the spirit of [15] may have been developed, but will not be considered here. We also refer to [7], for a recent work on this direction.

Our approach here consists in first considering the one dimensional linear advection (like in the splitting procedure) in order to analyse the stability properties of the two kinds of numerical schemes in a simplified framework. This information may be a good guide for the $2 \mathrm{D}$ context. We also make a link between finite volumes type approximation and semi-Lagrangian schemes. Indeed, when a Lagrange reconstruction is used in semi-Lagrangian schemes, we show that when the time step tends towards zero, we can recover some standard approximations (upwind) of the fluxes when a finite volumes approach is used. Others links can also be performed.

\section{Finite volumes method of Banks [4]}

This section is devoted to the presentation and analysis of a finite volumes method. The 1D case will be tackled together with a stability analysis. Then, we detail the 2D case.

\subsection{The 1D linear advection}

We first focus on solving the 1D linear advection problem:

$$
\left\{\begin{array}{l}
\frac{\partial f(t, x)}{\partial t}+a \frac{\partial f(t, x)}{\partial x}=0 \\
f(t=0, x)=f^{0}(x)
\end{array}\right.
$$

where $f:\left[0,+\infty\left[\times \Omega \longrightarrow \mathbb{R}\right.\right.$ and $a$ is a real constant. The unknowns are $\bar{f}_{i}^{n}=\frac{1}{\Delta x} \int_{x_{i-1 / 2}}^{x_{i+1 / 2}} f\left(t_{n}, x\right) d x$ and we write:

$$
\bar{f}_{i}(t)=\frac{1}{\Delta x} \int_{x_{i-1 / 2}}^{x_{i+1 / 2}} f(t, x) d x
$$

Integrating (2) over a control volume and dividing by its size $\Delta x$, we get:

$$
\frac{d \bar{f}_{i}(t)}{d t}=-\frac{1}{\Delta x} \int_{x_{i-1 / 2}}^{x_{i+1 / 2}} a \partial_{x} f d x=-\frac{a}{\Delta x}\left[f\left(t, x_{i+1 / 2}\right)-f\left(t, x_{i-1 / 2}\right)\right]
$$

The main goal is to give a sense to the fluxes $f\left(t, x_{i \pm 1 / 2}\right)$ for a given sequence $\left(\bar{f}_{i}(t)\right)_{i}$.

For this, we write classically

$$
f\left(t, x_{i+1 / 2}\right) \approx \sum_{j=r}^{s} a_{j} \bar{f}_{i+j}(t),
$$

The order conditions allow to determine the coefficients by solving the system

$$
\frac{1}{2^{k}}=\sum_{j=r}^{s} a_{j} \int_{j-1 / 2}^{j+1 / 2} x^{k} d x, k=0, \ldots, r-s .
$$

Note that we consider here periodic boundary conditions. 
The following space discretizations will be tested

$$
\begin{aligned}
C D 2: & f\left(t, x_{i+1 / 2}\right) & \approx \frac{1}{2}\left(\bar{f}_{i}(t)+\bar{f}_{i+1}(t)\right), \\
C D 4: & f\left(t, x_{i+1 / 2}\right) & \approx \frac{7}{12}\left(\bar{f}_{i}(t)+\bar{f}_{i+1}(t)\right)-\frac{1}{12}\left(\bar{f}_{i-1}(t)+\bar{f}_{i+2}(t)\right), \\
C D 6: & f\left(t, x_{i+1 / 2}\right) & \approx \frac{37}{60}\left(\bar{f}_{i}(t)+\bar{f}_{i+1}(t)\right)-\frac{8}{60}\left(\bar{f}_{i-1}(t)+\bar{f}_{i+2}(t)\right)+\frac{1}{60}\left(\bar{f}_{i-2}(t)+\bar{f}_{i+3}(t)\right), \\
U P 1(a<0): & f\left(t, x_{i+1 / 2}\right) & \approx \bar{f}_{i+1}(t), \\
U P 3(a<0): & f\left(t, x_{i+1 / 2}\right) & \approx \frac{1}{3} \bar{f}_{i}(t)+\frac{5}{6} \bar{f}_{i+1}(t)-\frac{1}{6} \bar{f}_{i+2}(t), \\
U P 5(a<0): & f\left(t, x_{i+1 / 2}\right) & \approx-\frac{1}{20} \bar{f}_{i-1}(t)+\frac{9}{20} \bar{f}_{i}(t)+\frac{47}{60} \bar{f}_{i+1}(t)-\frac{13}{60} \bar{f}_{i+2}(t)+\frac{1}{30} \bar{f}_{i+3}(t), \\
U P 1(a>0): & f\left(t, x_{i+1 / 2}\right) & \approx \bar{f}_{i}(t), \\
U P 3(a>0): & f\left(t, x_{i+1 / 2}\right) & \approx-\frac{1}{6} \bar{f}_{i-1}(t)+\frac{5}{6} \bar{f}_{i}(t)+\frac{1}{3} \bar{f}_{i+1}(t), \\
U P 5(a>0): & f\left(t, x_{i+1 / 2}\right) & \approx \frac{1}{30} \bar{f}_{i-2}(t)-\frac{13}{60} \bar{f}_{i-1}(t)+\frac{47}{60} \bar{f}_{i}(t)+\frac{9}{20} \bar{f}_{i+1}(t)-\frac{1}{20} \bar{f}_{i+2}(t) .
\end{aligned}
$$

A classical time discretization with an explicit Runge-Kutta algorithm is then used and leads to the computation of

$$
\bar{f}^{n}=\left(\bar{f}_{0}^{n}, \ldots, \bar{f}_{N-1}^{n}\right), f_{j}^{n} \simeq f_{j}\left(t_{n}\right), j=0, \ldots, N-1 .
$$

More precisely, the numerical approximation $y_{n} \approx y\left(t_{n}\right)$ of the differential system obtained from (3) written in the form $y^{\prime}(t)=\phi(y(t))$ is given by

$$
y_{n+1} \approx y_{n}+\Delta t \sum_{j=1}^{s} b_{j} k_{j}, k_{j}=\phi\left(y_{n}+\Delta t \sum_{\ell=1}^{j-1} a_{j, \ell} k_{\ell}\right), j=1, \ldots, s,
$$

and we have considered the following examples

$$
\begin{array}{ll}
R K 1(s=1) & b_{1}=1, \\
R K 2(s=2) & a_{2,1}=1 / 2, b_{1}=0, b_{2}=1, \\
R K 3(s=3) & a_{2,1}=1 / 2, a_{3,1}=-1, a_{3,2}=2, b_{1}=1 / 6, b_{2}=2 / 3, b_{3}=1 / 6 .
\end{array}
$$

and the classical RK4 scheme

$$
R K 4(s=4) \quad a_{2,1}=1 / 2, a_{3,1}=0, a_{3,2}=1 / 2, a_{4,1}=a_{4,2}=0, a_{4,3}=1, b_{1}=b_{4}=1 / 6, b_{2}=b_{3}=1 / 3 .
$$

\subsection{Stability and order}

We first tried to find out a superior limit above which these schemes are unstable, that is to determine a CFL condition for all these schemes. We can see in Table 1 which CFLs we found (see also [2]).

An example of such a computation is now given in the case CD4 RK1 (Euler). We perform a Von Neumann stability analysis. For this, we introduce

$$
f_{j}^{n}=\sum_{k=0}^{N-1} \hat{f_{k}^{n}} \exp (i k j \Delta x), \quad \text { with } \quad \hat{f}_{j}^{n}=\frac{1}{N} \sum_{k=0}^{N-1} f_{k}^{n} \exp (-i k j \Delta x),
$$




\begin{tabular}{|c|c|c|c|c|c|c|}
\hline & up 1 & CD 2 & up 3 & CD 4 & up 5 & CD 6 \\
\hline RK 1 & 1.00 & 0.00 & 0.00 & 0.00 & 0.00 & 0.00 \\
\hline RK 2 & 1.00 & 0.00 & 0.87 & 0.00 & 0.00 & 0.00 \\
\hline RK 3 & 1.25 & 1.73 & 1.62 & 1.26 & 1.43 & 1.09 \\
\hline RK 4 & 1.39 & 2.82 & 1.74 & 2.06 & 1.73 & 1.78 \\
\hline
\end{tabular}

TABLE 1. CFL conditions for the finite volumes schemes.

so that $\hat{f}_{k+p}^{n}=\hat{f}_{k}^{n} e^{i k p \Delta x}$. The numerical scheme then becomes in the Fourier space

$$
\hat{f}_{k}^{n+1}=h_{k} \hat{f}_{k}^{n}, \text { with } h_{k}=1-\frac{a \Delta t}{6 \Delta x} i(6 \sin (k \Delta x)-\sin (2 k \Delta x)) .
$$

As for $\Delta t>0$ and $k \neq 0$, the amplification factor $\left|h_{k}\right|$ is strictly greater than 1 , we see that this scheme is unconditionally unstable.

It is worth mentioning that the use of high order Runge-Kutta schemes enables to overcome this lack of stability of low order Runge-Kutta scheme (see [2]). Note that the RK2 scheme is unstable, which is generally not the case for semi-lagrangian schemes.

Considering the stable cases CD2 RK4 and CD4 RK4, we look for determining numerically the order for the method. We choose the periodic initial condition $f^{0}(x)=\sin (2 \pi x)$ over the $1 \mathrm{D}$ domain $[0,1]$, with the following parameters:

$$
\left\{\begin{array}{l}
a=1 \\
\Delta t=0.001 \\
t_{\max }=16
\end{array}\right.
$$

In Figure 1, we plot in red the error in the $L^{1}$ norm of the reconstruction obtained for different numbers of points in space with the CD2 RK4 method (left) and the CD4 RK4 method (right). Thus, we see that the corresponding order are recovered: CD2 RK4 is second order and CD4 RK4 is fourth order.
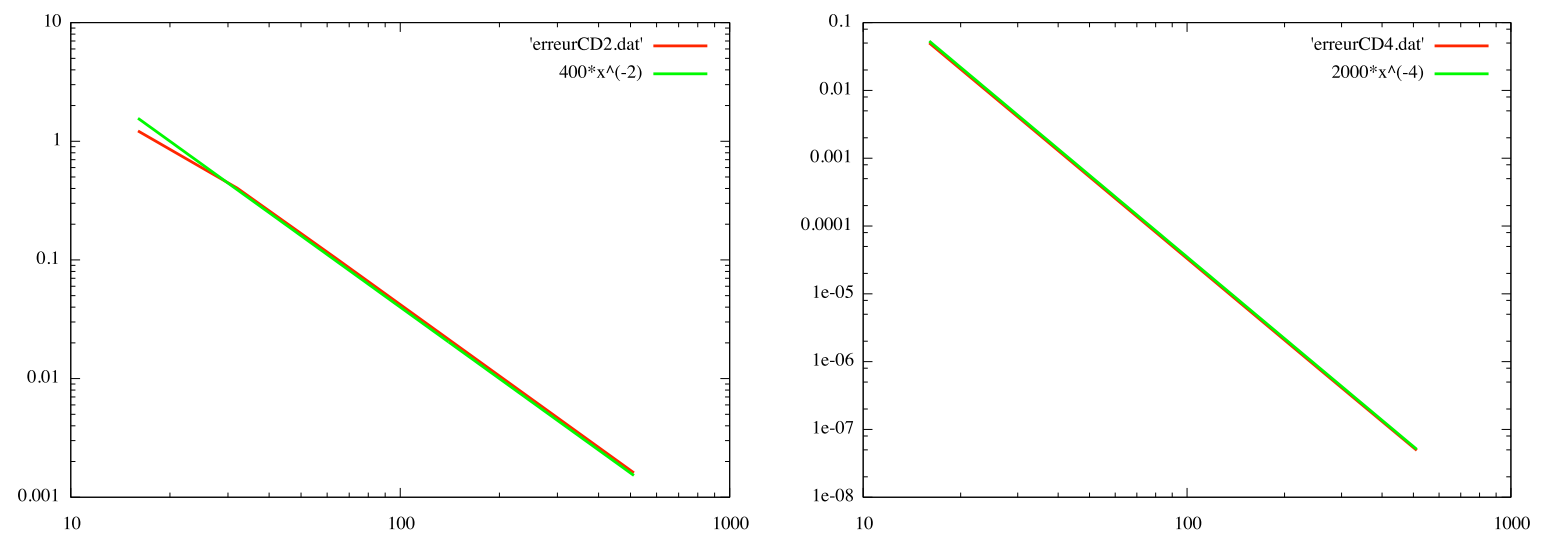

FIGURE 1. $L^{1}$ norm of the error for the linear advection as a function of $N_{x}$ with (left) CD2 RK4 scheme and (right) CD4 RK4 scheme. $\Delta t=0.001$ and $t_{\max }=16$. 


\section{3. $2 \mathrm{D}$ advection}

The extension to the 2D case is detailed here in view of applications to the Vlasov-Poisson system. The general model we have in mind is

$$
\partial_{t} f(t, x, y)+\partial_{x}\left(a_{x}(t, x, y) f(t, x, y)\right)+\partial_{y}\left(a_{y}(t, x, y) f(t, x, y)\right)=0,
$$

with $a=\left(a_{x}, a_{y}\right)$ a vector field which satisfies the divergence condition $\nabla \cdot a=\partial_{x} a_{x}+\partial_{y} a_{y}=0$. The unknowns are then

$$
\bar{f}_{i, j}^{n}=\frac{1}{\Delta x \Delta y} \int_{x_{i-1 / 2}}^{x_{i+1 / 2}} \int_{y_{j-1 / 2}}^{y_{j+1 / 2}} f\left(t_{n}, x, y\right) d x d y .
$$

To focus on the spatial discretization, we introduce

$$
\bar{f}_{i, j}(t)=\frac{1}{\Delta x \Delta y} \int_{x_{i-1 / 2}}^{x_{i+1 / 2}} \int_{y_{j-1 / 2}}^{y_{j+1 / 2}} f(t, x, y) d x d y
$$

Integrating (6) over a control volume and dividing by its size $\Delta x \Delta y$, we get:

$$
\begin{aligned}
\frac{d \bar{f}_{i, j}(t)}{d t}=-\frac{1}{\Delta x \Delta y} \int_{y_{j-1 / 2}}^{y_{j+1 / 2}}\left[a_{x}\left(t, x_{i+1 / 2}, y\right) f\left(t, x_{i+1 / 2}, y\right)-a_{x}\left(t, x_{i-1 / 2}, y\right) f\left(t, x_{i-1 / 2}, y\right)\right] d y \\
\quad-\frac{1}{\Delta x \Delta y} \int_{x_{i-1 / 2}}^{x_{i+1 / 2}}\left[a_{y}\left(t, x, y_{j+1 / 2}\right) f\left(t, x, y_{j+1 / 2}\right)-a_{y}\left(t, x, y_{j-1 / 2}\right) f\left(t, x, y_{j-1 / 2}\right)\right] d y .
\end{aligned}
$$

We next use a formula which permits to express the integral of the product in terms of product of integrals

Proposition 1.1. We have

$$
\begin{aligned}
\frac{1}{h} \int_{z_{i-1 / 2}}^{z_{i+1 / 2}} b(x) g(x) d x & =\frac{1}{h} \int_{z_{i-1 / 2}}^{z_{i+1 / 2}} b(x) d x \cdot \frac{1}{h} \int_{z_{i-1 / 2}}^{z_{i+1 / 2}} g(x) d x \\
+ & \frac{1}{48}\left(\int_{z_{i+1 / 2}}^{z_{i+3 / 2}} b(x) d x-\int_{z_{i-3 / 2}}^{z_{i-1 / 2}} b(x) d x\right)\left(\int_{z_{i+1 / 2}}^{z_{i+3 / 2}} g(x) d x-\int_{z_{i-3 / 2}}^{z_{i-1 / 2}} g(x) d x\right)+O\left(h^{4}\right) .
\end{aligned}
$$

Remark 1.2. Such formulae (and more general ones) are developed in $[4,11]$ and enable a high order space approximation.

For convenience, we shall give here a proof.

Proof of Proposition 1.1. We start from the well known midpoint approximation

$$
\frac{1}{h} \int_{z_{i-1 / 2}}^{z_{i+1 / 2}} f(x) d x=f\left(z_{i}\right)+\frac{h^{2}}{24} \partial_{x}^{2} f\left(z_{i}\right)+O\left(h^{4}\right)
$$

The left hand side gives

$$
b\left(z_{i}\right) g\left(z_{i}\right)+\frac{h^{2}}{24}\left(b^{\prime \prime}\left(z_{i}\right) g\left(z_{i}\right)+2 b^{\prime}\left(z_{i}\right) g^{\prime}\left(z_{i}\right)+b\left(z_{i}\right) g^{\prime \prime}\left(z_{i}\right)\right)+O\left(h^{4}\right),
$$


whereas the right hand side gives

$$
\begin{aligned}
&\left(b\left(z_{i}\right)+\frac{h^{2}}{24} b^{\prime \prime}\left(z_{i}\right)\right)\left(g\left(z_{i}\right)+\frac{h^{2}}{24} g^{\prime \prime}\left(z_{i}\right)\right)+O\left(h^{4}\right) \\
&+\frac{1}{48}\left(b\left(z_{i+1}\right)-b\left(z_{i-1}\right)+\frac{h^{2}}{24}\left(b^{\prime \prime}\left(z_{i+1}\right)-b^{\prime \prime}\left(z_{i-1}\right)\right)\right)\left(g\left(z_{i+1}\right)-g\left(z_{i-1}\right)+\frac{h^{2}}{24}\left(g^{\prime \prime}\left(z_{i+1}\right)-g^{\prime \prime}\left(z_{i-1}\right)\right)\right) \\
&=b\left(z_{i}\right) g\left(z_{i}\right)+\frac{h^{2}}{24} b^{\prime \prime}\left(z_{i}\right)\left(b^{\prime \prime}\left(z_{i}\right) g\left(z_{i}\right)+b\left(z_{i}\right) g^{\prime \prime}\left(z_{i}\right)\right)+O\left(h^{4}\right) \\
&+\frac{h^{2}}{48}\left(2 b^{\prime}\left(z_{i}\right)+O\left(h^{2}\right)\right)\left(2 g^{\prime}\left(z_{i}\right)+O\left(h^{2}\right)\right)
\end{aligned}
$$

which gives the result.

Finally, we proceed like in the 1D case. As an example, the CD4 approximation gives

$$
\frac{1}{\Delta y} \int_{y_{j-1 / 2}}^{y_{j+1 / 2}} f\left(t, x_{i+1 / 2}, y\right) d y \approx \frac{7}{12}\left(\bar{f}_{i, j}(t)+\bar{f}_{i+1, j}(t)\right)-\frac{1}{12}\left(\bar{f}_{i-1, j}(t)+\bar{f}_{i+2, j}(t)\right),
$$

We thus get a system of ODE's which is discretized in time with a Runge Kutta scheme like in the 1D case.

\subsection{Application to the Vlasov-Poisson system}

For the Vlasov-Poisson system, we have $a_{x}(t, x, v)=v$ and $a_{v}(t, x, v)=E(t, x)$ in (6). The electric field is computed using the charge density $\int f(t, x, v) d v$ that is recomputed after each step of the Runge-Kutta method.

\section{Gauss points in time methods}

The purpose of this section is to present a finite volume type method based on a semi-Lagrangian integration of the fluxes. The method is first presented in $1 \mathrm{D}$ for which a stability analysis is performed. Then the $2 \mathrm{D}$ case is focused on.

\subsection{The 1D linear advection}

Starting with the advection equation (3), the integration in time between $t^{n}$ and $t^{n+1}$ leads to the computation of $\int_{t^{n}}^{t^{n+1}} f\left(t, x_{i+1 / 2}\right) d t$. Thanks to the change of variables $t=t^{n}+\Delta t(1+s) / 2$ with $s \in[-1,1]$, a Gauss quadrature can be performed: introducing the $K$ Gauss points and weights $\left(\omega_{k}, \tau_{k}\right)$ for the interval $[-1,1]$ leads to

$$
\int_{t^{n}}^{t^{n+1}} f\left(t, x_{i+1 / 2}\right) d t \approx \frac{\Delta t}{2} \sum_{k=1}^{K} \omega_{k} f\left(t^{n}+\frac{\Delta t}{2}\left(1+\tau_{k}\right), x_{i+1 / 2}\right)
$$

Using the fact that $f$ is constant along the characteristics, the right hand side member can be expressed as function of $f\left(t^{n}\right)$

$$
\int_{t^{n}}^{t^{n+1}} f\left(t, x_{i+1 / 2}\right) d t \approx \frac{\Delta t}{2} \sum_{k=1}^{K} \omega_{k} f\left(t^{n}, x_{i+1 / 2}-a\left(\frac{\Delta t}{2}\left(1+\tau_{k}\right)\right)\right),
$$

so that the numerical scheme is

$$
\left.\left.\bar{f}_{i}^{n+1}=\bar{f}_{i}^{n}-\frac{a \Delta t}{2 \Delta x} \sum_{k=1}^{K} \omega_{k}\left[f\left(t^{n}, x_{i+1 / 2}-\frac{a \Delta t}{2}\left(1+\tau_{k}\right)\right)\right)-f\left(t^{n}, x_{i-1 / 2}-\frac{a \Delta t}{2}\left(1+\tau_{k}\right)\right)\right)\right] .
$$


The quantities $f\left(t^{n}, x_{i+1 / 2}-a \Delta t\left(1+\tau_{k}\right) / 2\right)$ have to be reconstructed using the known mean values $\bar{f}_{i}^{n}, \quad i=$ $0, \ldots, N-1$ using an interpolation operator. Some reconstructions will be detailed hereafter.

Remark 2.1. For instance, if we choose $N_{k}=1$ Gauss point, $\omega_{k}=2$ and $\tau_{k}=0$, we get the midpoint formula:

$$
\int_{t^{n}}^{t^{n+1}} f\left(t, x_{i+1 / 2}\right) d t \approx \Delta t f\left(t^{n}, x_{i+1 / 2}-a \frac{\Delta t}{2}\right) .
$$

If we choose $N_{k}=2$ Gauss points, $\omega_{1}=\omega_{2}=1, \tau_{1}=-1 / \sqrt{3}$ and $\tau_{2}=1 / \sqrt{3}$, we get:

$$
\int_{t^{n}}^{t^{n+1}} f\left(t, x_{i+1 / 2}\right) d t \approx \frac{\Delta t}{2}\left(f\left(t^{n}, x_{i+1 / 2}-a \frac{\Delta t(3-\sqrt{3})}{6}\right)+f\left(t^{n}, x_{i+1 / 2}-a \frac{\Delta t(3+\sqrt{3})}{6}\right)\right) .
$$

Remark 2.2. The introduction of Gauss points in time for the linear advection is not really useful, since we have the relation between time integration and space integration (see Section 3 for a proof)

$$
a \int_{t_{n}}^{t_{n+1}} f\left(t, x_{i+1 / 2}\right) d t=\int_{x_{i+1 / 2}^{*}}^{x_{i+1 / 2}} f\left(t_{n}, y\right) d y
$$

where $x_{i+1 / 2}^{*}=x_{i+1 / 2}-a \Delta t$ is the foot of the characteristic ending at $x_{i+1 / 2}$. Since the values $\bar{f}_{i}^{n}, i=0, \ldots, N-1$ are known, the right hand side can be approximated with an appropriate reconstruction as detailed after. In particular, there is then no CFL restriction. However the extension to the $2 D$ case involves the computation of the intersection between the Lagrangian volume and the Eulerian one, see [20]. The use of Gauss points avoids doing this technical step and is thus an alternative that we propose to explore here. Other strategies can also be envisaged (see [7, 15] where possible extensions of the 1D finite volumes to 2D unsplit schemes are detailed).

Reconstruction. The method has to be completed with a reconstruction operator to compute $f\left(t^{n}, x_{i+1 / 2}-\right.$ $\left.a \Delta t\left(1+\tau_{k}\right) / 2\right)$. A lot of interpolation operators can be considered to achieve this task [10], [13], [18]. We look for a polynomial $P_{i}$ on each cell $\left[x_{i-1 / 2}, x_{i+1 / 2}\right]$ which satisfies

$$
\frac{1}{\Delta x} \int_{x_{i-1 / 2}}^{x_{i+1 / 2}} P_{i}(x) d x=\bar{f}_{i}^{n}
$$

Lagrange reconstructions (LAG-2d+1) consist in taking $P_{i}$ of degree $\leq 2 d$ satisfying the constraints

$$
\frac{1}{\Delta x} \int_{x_{j-1 / 2}}^{x_{j+1 / 2}} P_{i}(x) d x=\bar{f}_{i}^{n}, j=i-d, \ldots, i+d .
$$

PPM like reconstructions consist in taking $P_{i}$ of degree $\leq 2$ satisfying (12) together with

$$
P_{i}\left(x_{i-1 / 2}\right)=f_{i-1 / 2}^{n}, P_{i}\left(x_{i+1 / 2}\right)=f_{i+1 / 2}^{n},
$$

that is

$$
P_{i}\left(x_{i-1 / 2}+\alpha \Delta x\right)=\left(3 \alpha^{2}-4 \alpha+1\right) f_{i-1 / 2}^{n}+\left(3 \alpha^{2}-2 \alpha\right) f_{i+1 / 2}^{n}+\left(6 \alpha-6 \alpha^{2}\right) \bar{f}_{i}^{n} \quad \text { with } \alpha \in[0,1] .
$$




\begin{tabular}{|c|c|c|c|c|c|c|c|}
\hline$K$ & LAG-1 & LAG-3 & LAG-5 & LAG-7 & PPM 0 & PPM 1 & PPM 2 \\
\hline 1 & 1.00 & 0.68 & 0.00 & 0.00 & 0.72 & 0.66 & 0.66 \\
\hline 2 & 1.00 & 1.00 & 1.00 & 1.00 & 1.63 & 1.70 & 1.73 \\
\hline 3 & 1.00 & 1.00 & 1.00 & 1.00 & 2.00 & 1.54 & 1.54 \\
\hline 4 & 1.00 & 1.00 & 1.00 & 1.00 & 1.77 & 1.83 & 1.88 \\
\hline 5 & 1.85 & 2.00 & 2.00 & 2.00 & 2.69 & 2.69 & 2.69 \\
\hline
\end{tabular}

TABLE 2. CFL conditions with $K$ Gauss points

The interface values $f_{i+1 / 2}^{n} \approx f\left(t^{n}, x_{i+1 / 2}\right)$ are given by

$$
\begin{array}{lll}
\text { PPM0 : } & f_{i+1 / 2}^{n}=\frac{1}{2}\left(\bar{f}_{i}^{n}+\bar{f}_{i+1}^{n}\right), \\
\text { PPM1: } & f_{i+1 / 2}^{n}=\frac{7}{12}\left(\bar{f}_{i}^{n}+\bar{f}_{i+1}^{n}\right)-\frac{1}{12}\left(\bar{f}_{i-1}^{n}+\bar{f}_{i+2}^{n}\right), \\
\text { PPM2: } & f_{i+1 / 2}^{n}=\frac{37}{60}\left(\bar{f}_{i}^{n}+\bar{f}_{i+1}^{n}\right)-\frac{8}{60}\left(\bar{f}_{i-1}^{n}+\bar{f}_{i+2}^{n}\right)+\frac{1}{60}\left(\bar{f}_{i-2}^{n}+\bar{f}_{i+3}^{n}\right) .
\end{array}
$$

Remark 2.3. The notation $L A G-2 d+1$ could be strange, since we deal with polynomial of degree $\leq 2 d$. However, if we consider the reconstruction without the Gauss points approximation, as explained in Remark 2.2, we can see that this method is equivalent to the pointwise semi-Lagrangian scheme with Lagrange interpolation of degree $\leq 2 d+1$ (see [13]).

\section{Stability analysis and order}

As in subsection 1.2, we find the CFL conditions, by studying numerically the amplification factor. The results are given in Table 2.

On Figure 2, the spatial error in $L^{1}$ norm is plotted for a constant advection case (with a Gaussian initial condition). In the case of 2 Gauss points in time, we see that the orders are recovered: LAG-3 is order 3 and LAG- 5 is order 5 .
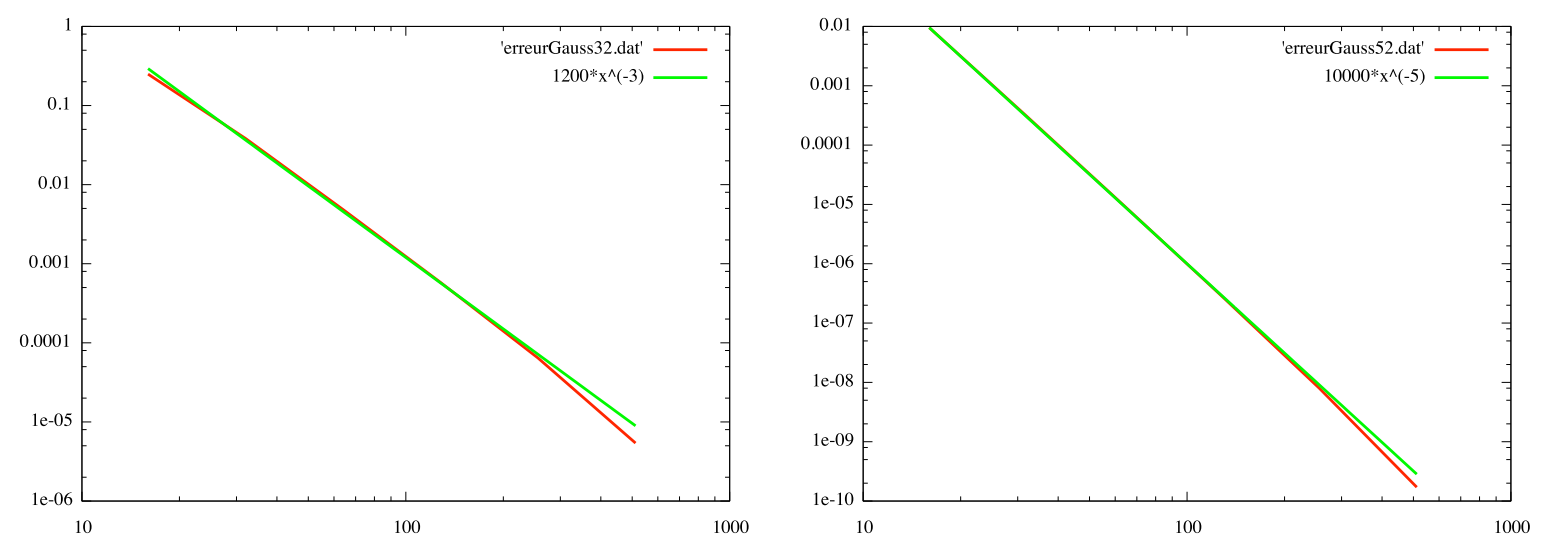

Figure 2. $L^{1}$ norm of the error for the linear advection as a function of $N_{x}$ with (left) Lag-3 reconstruction and (right) Lag-5 reconstruction. $\Delta t=0.001$ and $t_{\max }=16$. 


\section{2. $2 \mathrm{D}$ advection}

The extension to the 2D case is discussed here. As previously, we integrate over a control volume and divide it by its size $\Delta x \Delta y$, and we get the semi-discrete scheme

$$
\begin{aligned}
\frac{d \bar{f}_{i, j}(t)}{d t}=-\frac{1}{\Delta x \Delta y} \int_{y_{j-1 / 2}}^{y_{j+1 / 2}}\left(a_{x}\left(t, x_{i+1 / 2}, y\right) f\left(t, x_{i+1 / 2}, y\right)-a_{x}\left(t, x_{i-1 / 2}, y\right) f\left(t, x_{i-1 / 2}, y\right)\right) d y & \\
- & \frac{1}{\Delta x \Delta y} \int_{x_{i-1 / 2}}^{x_{i+1 / 2}}\left(a_{y}\left(t, x, y_{j+1 / 2}\right) f\left(t, x, y_{j+1 / 2}\right)-a_{y}\left(t, x, y_{j-1 / 2}\right) f\left(t, x, y_{j-1 / 2}\right)\right) d y
\end{aligned}
$$

To compute the two integrals, we here introduce Gauss points in space: $d_{x}$ points $\left(\omega_{x, \ell}, \tau_{x, \ell}\right)$ for the $x$-direction, and $d_{y}$ points $\left(\omega_{y, \ell}, \tau_{y, \ell}\right)$ for the $y$-direction

$$
\begin{aligned}
& \frac{d \bar{f}_{i, j}(t)}{d t} \approx- \frac{1}{2 \Delta x} \sum_{\ell=1}^{d_{y}} \omega_{y, \ell}\left[a_{x}\left(t, x_{i+1 / 2}, y_{j}+\frac{\Delta y}{2}\left(1+\tau_{y, \ell}\right)\right) f\left(t, x_{i+1 / 2}, y_{j}+\frac{\Delta y}{2}\left(1+\tau_{y, \ell}\right)\right)\right. \\
&\left.-a_{x}\left(t, x_{i-1 / 2}, y_{j}+\frac{\Delta y}{2}\left(1+\tau_{y, \ell}\right)\right) f\left(t, x_{i-1 / 2}, y_{j}+\frac{\Delta y}{2}\left(1+\tau_{y, \ell}\right)\right)\right] \\
&-\frac{1}{2 \Delta y} \sum_{\ell=1}^{d_{x}} \omega_{x, \ell}\left[a_{y}\left(t, x_{i}+\frac{\Delta x}{2}\left(1+\tau_{x, \ell}\right), y_{j+1 / 2}\right) f\left(t, x_{i}+\frac{\Delta x}{2}\left(1+\tau_{x, \ell}\right), y_{j+1 / 2}\right)\right. \\
&\left.-a_{y}\left(t, x_{i}+\frac{\Delta x}{2}\left(1+\tau_{x, \ell}\right), y_{j-1 / 2}\right) f\left(t, x_{i}+\frac{\Delta x}{2}\left(1+\tau_{x, \ell}\right), y_{j-1 / 2}\right)\right]
\end{aligned}
$$

Now, we apply the same strategy as in the $1 \mathrm{D}$ case: using $K$ Gauss points in time $\left(\omega_{k}, \tau_{k}\right)$ for the quadrature in time, we then obtain

$$
\begin{gathered}
\bar{f}_{i, j}^{n+1} \approx \bar{f}_{i, j}^{n}-\frac{\Delta t}{4 \Delta x} \sum_{k=1}^{K} \sum_{\ell=1}^{d_{y}} \omega_{k} \omega_{y, \ell}\left[a_{x}\left(t^{n}+\frac{\Delta t}{2}\left(1+\tau_{k}\right), x_{i+1 / 2}, y_{j}+\frac{\Delta y}{2}\left(1+\tau_{y, \ell}\right)\right) f\left(t^{n},\left(x_{i+1 / 2}, y_{j}+\frac{\Delta y}{2}\left(1+\tau_{y, \ell}\right)\right)^{*_{k}}\right)\right. \\
\left.-a_{x}\left(t^{n}+\frac{\Delta t}{2}\left(1+\tau_{k}\right), x_{i-1 / 2}, y_{j}+\frac{\Delta y}{2}\left(1+\tau_{y, \ell}\right)\right) f\left(t^{n},\left(x_{i-1 / 2}, y_{j}+\frac{\Delta y}{2}\left(1+\tau_{y, \ell}\right)\right)^{*_{k}}\right)\right] \\
-\frac{\Delta t}{4 \Delta y} \sum_{k=1}^{K} \sum_{\ell=1}^{d_{x}} \omega_{k} \omega_{x, \ell}\left[a_{y}\left(t^{n}+\frac{\Delta t}{2}\left(1+\tau_{k}\right), x_{i}+\frac{\Delta x}{2}\left(1+\tau_{x, \ell}\right), y_{j+1 / 2}\right) f\left(t^{n},\left(x_{i}+\frac{\Delta x}{2}\left(1+\tau_{x, \ell}\right), y_{j+1 / 2}\right)^{*_{k}}\right)\right. \\
\left.-a_{y}\left(t^{n}+\frac{\Delta t}{2}\left(1+\tau_{k}\right), x_{i}+\frac{\Delta x}{2}\left(1+\tau_{x, \ell}\right), y_{j-1 / 2}\right) f\left(t^{n},\left(x_{i}+\frac{\Delta x}{2}\left(1+\tau_{x, \ell}\right), y_{j-1 / 2}\right)^{*_{k}}\right)\right],
\end{gathered}
$$

where $(x, y)^{*_{k}}$ denotes the feet at time $t^{n}$ of the characteristic ending at $(x, y)$ at time $t^{n}+\Delta t\left(1+\tau_{k}\right) / 2$. By using a predictor corrector scheme for example, we will suppose that the fields $a_{x}$ and $a_{y}$ are constant over the time domain $\left[t^{n}, t^{n+1}\right]: a_{x}(t, x, y) \simeq a_{x}\left(t^{n+1 / 2}, x, y\right)$ and $a_{y}(t, x, y) \simeq a_{y}\left(t^{n+1 / 2} x, y\right)$ for all $t \in\left[t^{n}, t^{n+1}\right]$, and the fields $a_{x}\left(t^{n+1 / 2}, x, y\right)$ and $a_{y}\left(t^{n+1 / 2}, x, y\right)$ are predicted with a suitable method. 
Remark 2.4. The case of one Gauss point in space will be essentially used. The numerical scheme writes

$$
\begin{gathered}
\bar{f}_{i, j}^{n+1} \approx \bar{f}_{i, j}^{n}-\frac{\Delta t}{2 \Delta x} \sum_{k=1}^{K} \omega_{k}\left[a_{x}\left(t^{n+1 / 2}, x_{i+1 / 2}, y_{j}\right) f\left(t^{n},\left(x_{i+1 / 2}, y_{j}\right)^{*_{k}}\right)-a_{x}\left(t^{n+1 / 2}, x_{i-1 / 2}, y_{j}\right) f\left(t^{n},\left(x_{i-1 / 2}, y_{j}\right)^{*_{k}}\right)\right] \\
-\frac{\Delta t}{2 \Delta y} \sum_{k=1}^{K} \omega_{k}\left[a_{y}\left(t^{n+1 / 2}, x_{i}, y_{j+1 / 2}\right) f\left(t^{n},\left(x_{i}, y_{j+1 / 2}\right)^{*_{k}}\right)-a_{y}\left(t^{n+1 / 2}, x_{i}, y_{j-1 / 2}\right) f\left(t^{n},\left(x_{i}, y_{j-1 / 2}\right)^{*_{k}}\right)\right],
\end{gathered}
$$

where $\left(x_{i+1 / 2}, y_{j}\right)^{*_{k}}$ denote the feet at time $t^{n}$ of the characteristics ending at $\left(x_{i+1 / 2}, y_{j}\right)$ at time $t^{n}+\Delta t(1+$ $\left.\tau_{k}\right) / 2$.

\subsection{Application to the Vlasov-Poisson case}

We will now focus on the Vlasov-Poisson equation which corresponds to $a_{x}(t, x, v)=v$ and $a_{y}(t, x, v)=$ $E(t, x)$.

In this case, (13) can be simplified into

$$
\begin{aligned}
\bar{f}_{i, j}^{n+1} \approx \bar{f}_{i, j}^{n}-\frac{\Delta t v_{j}}{2 \Delta x} \sum_{k=1}^{K} \omega_{k}\left[f\left(t^{n},\left(x_{i+1 / 2}, v_{j}\right)^{*_{k}}\right)-f\left(t^{n},\left(x_{i-1 / 2}, v_{j}\right)^{*_{k}}\right)\right] \\
-\frac{\Delta t E\left(t^{n+1 / 2}, x_{i}\right)}{2 \Delta v} \sum_{k=1}^{K} \omega_{k}\left[f\left(t^{n},\left(x_{i}, v_{j+1 / 2}\right)^{*_{k}}\right)-f\left(t^{n},\left(x_{i}, v_{j-1 / 2}\right)^{*_{k}}\right)\right]
\end{aligned}
$$

Prediction of $E\left(t^{n+1 / 2}\right)$. The electric field $E\left(t^{n+1 / 2}\right)$ is approximated by a prediction: we compute $\bar{f}_{i, j}^{n+1 / 2}$ by using the scheme with $\Delta t / 2$ instead of $\Delta t$ and we take the electric field using the charge density at time $t^{n}$. This enables to compute the charge density and thus the approximation of $E\left(t^{n+1 / 2}\right)$ by using $\bar{f}_{i, j}^{n+1 / 2}$, which is used for the correction step.

Computation of the characteristics A Verlet scheme is used for the computation of the characteristics: by writing for example $\left(X^{n+1}, V^{n+1}\right)=\left(x_{i+1 / 2}, v_{j}\right)$ and $\left(X^{n}, V^{n}\right)=\left(x_{i+1 / 2}, v_{j}\right)^{*_{k}}$, we have

$$
\left\{\begin{array}{l}
X^{n+1 / 2}=X^{n+1}-\frac{\Delta t}{2} V^{n+1} \\
V^{n}=V^{n+1}-\Delta t E\left(X^{n+1 / 2}\right) \\
X^{n}=X^{n+1 / 2}-\frac{\Delta t}{2} V^{n}
\end{array}\right.
$$

where $E$ corresponds either to $E\left(t^{n}\right)$ (prediction step) or to $E\left(t^{n+1 / 2}\right)$ (correction step).

$2 \mathrm{D}$ reconstruction. The $2 \mathrm{D}$ reconstruction that is needed here consists in using a tensor product of $1 \mathrm{D}$ reconstructions.

Remark 2.5. We found out that all PPM methods are unstable in the $2 D$ case. We can perform the Von Neumann analysis for $a_{x}=1, a_{y}=0$ and CFL number $A=\Delta t / \Delta x$. We use the initial condition $\exp \left(i k_{x} x\right) \exp \left(i k_{y} y\right)$. Choosing $k_{x}=\pi / 6$ and $k_{y}=4 \pi / 6$, we found that the amplification factor $h_{k_{x}, k_{y}}$ satisfies the formula

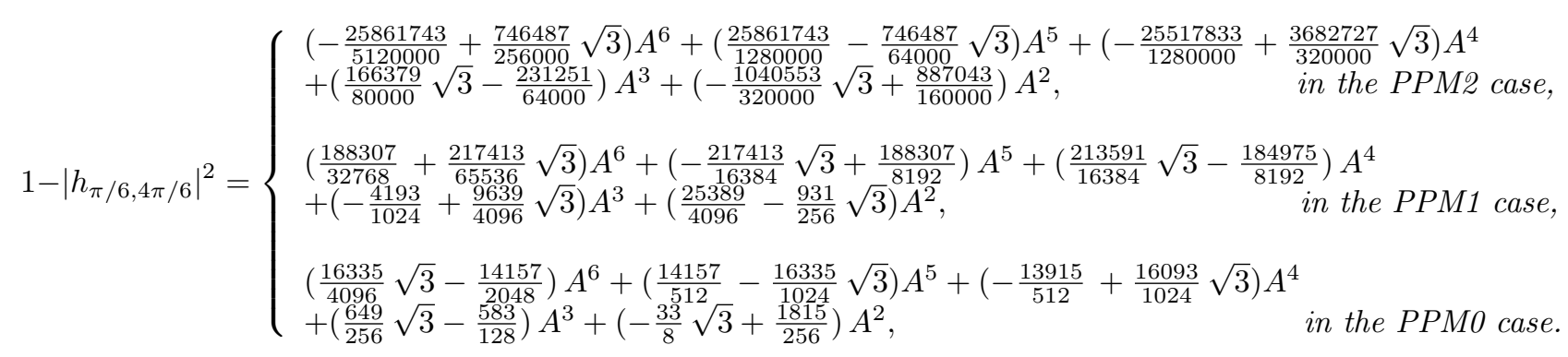


Since the leading term in $A^{2}$ is always negative, there exists $A_{0}>0$ such that for $0<A<A_{0}$, the method is unstable. Note that this phenomenon did not appear in the $1 D$ case.

\section{LiNKS BETWEEN FINITE VOLUMES AND SEMI-LAGRANGIAN SCHEMES}

We first establish the identity (11), which makes the link between the finite volumes and the semi-Lagrangian form of the flux. This result, valid for general field $a(t, x)$, was already proved in [22] for example, by using the Divergence Theorem. We give here another proof.

Proposition 3.1. We have

$$
\int_{t_{n}}^{t_{n+1}} a\left(t, x_{i+1 / 2}\right) f\left(t, x_{i+1 / 2}\right) d t=\int_{x_{i+1 / 2}^{*}}^{x_{i+1 / 2}} f\left(t_{n}, y\right) d y
$$

where

$$
\partial_{t} f(t, x)+\partial_{x}(a(t, x) f(t, x))=0, X^{\prime}(t)=a(t, X(t)), X\left(t_{n+1}\right)=x_{i+1 / 2}, X\left(t_{n}\right)=x_{i+1 / 2}^{*} .
$$

Proof. We write $X(t, s, x)$ the characteristic satisfying $\partial_{t} X(t, s, x)=a(t, X(t, s, x)), X(s, s, x)=x$. We have at first, following [17]

$$
\int_{t_{n}}^{t_{n+1}} a\left(t, x_{i+1 / 2}\right) f\left(t, x_{i+1 / 2}\right) d t=\int_{t_{n}}^{t_{n+1}} a\left(t, x_{i+1 / 2}\right) f\left(t_{n}, X\left(t_{n}, t, x_{i+1 / 2}\right)\right) \partial_{x} X\left(t_{n}, t, x_{i+1 / 2}\right) d t .
$$

We then make the change of variable $y=X\left(t_{n}, t, x_{i+1 / 2}\right)$, in order to pass from the time integral to the space integral. Note that we have

$$
X\left(t_{n}, t^{\prime}, X\left(t^{\prime}, t, x_{i+1 / 2}\right)\right)=X\left(t_{n}, t, x_{i+1 / 2}\right), \forall t^{\prime},
$$

which means that this quantity does not depend on $t^{\prime}$. The derivative against $t^{\prime}$ is thus zero, which means that

$$
\partial_{s} X\left(t_{n}, t^{\prime}, X\left(t^{\prime}, t, x_{i+1 / 2}\right)\right)+\partial_{t} X\left(t^{\prime}, t, x_{i+1 / 2}\right) \partial_{x} X\left(t_{n}, t^{\prime}, X\left(t^{\prime}, t, x_{i+1 / 2}\right)\right)=0
$$

that is

$$
\partial_{s} X\left(t_{n}, t^{\prime}, X\left(t^{\prime}, t, x_{i+1 / 2}\right)\right)=-a\left(t, X\left(t^{\prime}, t, x_{i+1 / 2}\right)\right) \partial_{x} X\left(t_{n}, t^{\prime}, X\left(t^{\prime}, t, x_{i+1 / 2}\right)\right) .
$$

By taking $t^{\prime}=t$, we get

$$
\partial_{s} X\left(t_{n}, t, x_{i+1 / 2}\right)=-a\left(t, x_{i+1 / 2}\right) \partial_{x} X\left(t_{n}, t, x_{i+1 / 2}\right),
$$

and thus $d y=-a\left(t, x_{i+1 / 2}\right) \partial_{x} X\left(t_{n}, t, x_{i+1 / 2}\right) d t$. As we have $X\left(t_{n}, t_{n}, x_{i+1 / 2}\right)=x_{i+1 / 2}$ and $X\left(t_{n}, t_{n+1}, x_{i+1 / 2}\right)=$ $x_{i+1 / 2}^{*}$, we get

$$
\int_{t_{n}}^{t_{n+1}} a\left(t, x_{i+1 / 2}\right) f\left(t, x_{i+1 / 2}\right) d t=-\int_{x_{i+1 / 2}}^{x_{i+1 / 2}^{*}} f\left(t_{n}, y\right) d y
$$

which gives the result. We refer to [5] for such computations on the characteristics.

Exponential integrator. We now make a link between the system of ODEs (the method of lines) arising from the finite volumes form (Section 1) and the semi-Lagrangian scheme (the limit where the number of Gauss points tends to infinity of Section 2) for a given discretization in space. We consider the constant advection problem. We have the following proposition 
Proposition 3.2. Consider the semi-Lagrangian scheme with a $L A G-2 d+1$ reconstruction applied $M$ times with time step $\Delta t / M$ which can be written in the form

$$
\left(f_{j}^{n+1, M}\right)_{j=0, \ldots, N-1}=\prod_{k=1}^{M} \mathcal{T}_{\Delta t / M}\left(f_{j}^{n, M}\right)_{j=0, \ldots, N-1} .
$$

We then have

$$
\lim _{M \rightarrow \infty} f_{j}^{n, M}=\bar{f}_{j}\left(t_{n}\right),, j=0, \ldots, N-1,
$$

where $\left(\bar{f}_{j}\right)_{j=0, \ldots, N-1}$ solves (3) by taking the upwind approximation UP-2d +1 (4) with $s=-r=d$ (for $a>0$ ).

Proof. Considering at first the semi discrete system of the finite volumes method, we have

$$
\frac{d \bar{f}_{i}}{d t}=-a\left(f_{i+1 / 2}-f_{i-1 / 2}\right), a>0,
$$

where the fluxes are approximated by an upwind scheme $f\left(t, x_{i+1 / 2}\right) \approx \sum_{j=-d}^{d} a_{j} \bar{f}_{i+j}(t)$ where the coefficients satisfy

$$
\frac{1}{2^{k}}=\sum_{j=-d}^{d} a_{j} \int_{j-1 / 2}^{j+1 / 2} x^{k} d x, k=0, \ldots, 2 d .
$$

The solution of the system of ODE (exponential integrator) can be viewed as a Euler approximation in time using the time step $\Delta / M$ and looking at the limit $M \rightarrow+\infty$. This can be performed easily using Von Neumann analysis. Indeed, with $f_{i+1 / 2}=\sum_{j=-d}^{d} c_{j} \bar{f}_{i+j}^{n}$, we then have $\left(\widehat{f}_{i+1 / 2}\right)_{k}=\sum_{j=-d}^{d} c_{j} \widehat{\bar{f}}_{k}^{n} e^{i k j \Delta x}$ and

$$
\left(\widehat{f}^{n+1}\right)_{k}=\widehat{\widehat{f}}_{k}^{n}\left(1-\nu\left(\sum_{j=-d}^{d} c_{j}\left(e^{i j k \Delta x}-e^{i(j-1) k \Delta x}\right)\right)\right)=\widehat{f}_{k}^{n}(1-\nu h(k)),
$$

where $\nu=a \Delta t /(M \Delta x)$ and $h(k)$ denotes the Fourier transform of the fluxes.

We then have

$$
\begin{aligned}
\lim _{M \rightarrow+\infty}(1-\nu h(k))^{M} & =\lim _{\substack{M \rightarrow+\infty\\
}} \exp (M \ln (1-\nu h(k))) \\
& \lim _{M \rightarrow+\infty} \exp (-M \nu h(k)) \\
& =\exp (-a \Delta t / \Delta x h(k)) .
\end{aligned}
$$

We then observe that the integrator is given by the exponential of $-a \Delta t / \Delta x$ times the Fourier transform of the fluxes.

On the other side, we consider the semi-Lagrangian method

$$
\bar{f}_{i}^{n+1}=\frac{1}{\Delta x} \int_{x_{i-1 / 2}-a \Delta t / M}^{x_{i+1 / 2}-a \Delta t / M} f\left(t^{n}, x\right) d x,
$$

where $f\left(t^{n}, x\right)$ is reconstructed by a polynomial function $p_{i}$ of degree $2 d$ satisfying the constraints

$$
\frac{1}{\Delta x} \int_{x_{i-1 / 2+j}}^{x_{i+1 / 2+j}} p_{i}(x) d x=\bar{f}_{i+j}^{n}=\frac{1}{\Delta x}\left(P_{i}\left(x_{i+1 / 2+j}\right)-P_{i}\left(x_{i-1 / 2+j}\right)\right), j=-d, \ldots, d,
$$


where $P_{i}$ denotes a primitive of $p_{i}$. Hence, the numerical scheme also writes, in terms of $P_{i}$

$$
\bar{f}_{i}^{n+1}=\frac{1}{\Delta x}\left(P_{i}\left(x_{i+1 / 2}-a \Delta t / M\right)-P_{i}\left(x_{i-1 / 2}-a \Delta t / N\right)\right),
$$

where we supposed $a>0$ and $a \Delta t / M<\Delta x$. With a Taylor expansion of $P_{i}\left(x_{i+1 / 2}-a \Delta t / M\right)$, we get

$$
\bar{f}_{i}^{n+1}=\bar{f}_{i}^{n}-\nu\left(p_{i}\left(x_{i+1 / 2}\right)-p_{i}\left(x_{i-1 / 2}\right)\right)+O\left(\nu^{2}\right)
$$

with $\nu=a \Delta t /(M \Delta x)$. A Von Neuman analysis leads to $\left(\widehat{\bar{f}}^{n+1}\right)_{k}=\left(\widehat{\bar{f}}^{n}\right)_{k}\left(1-\nu h(k)+O\left(\nu^{2}\right)\right)$ where $h(k) \widehat{\bar{f}}_{k}^{n}$ denotes the Fourier transform of $\left[p_{i}\left(x_{i+1 / 2}\right)-p_{i}\left(x_{i-1 / 2}\right)\right]$. Then, regarding the limit $M \rightarrow+\infty$ leads to

$$
\lim _{M \rightarrow+\infty}\left(1-\nu h(k)+O\left(\nu^{2}\right)\right)^{M}=\lim _{M \rightarrow+\infty} \exp \left(M \ln \left(1-\nu h(k)+O\left(\nu^{2}\right)\right)\right)=\exp (-a \Delta t / \Delta x h(k)) .
$$

Then it is sufficient to prove that $p_{i}\left(x_{i+1 / 2}\right)$ (in the conservative method) is equal to the approximation of $f\left(x_{i+1 / 2}\right)$ (in the finite volumes method). The value $p_{i}\left(x_{i+1 / 2}\right)$ can be written as $p_{i}\left(x_{i+1 / 2}\right)=\sum_{j=-d}^{d} a_{j} \bar{f}_{i+j}^{n}$ where $a_{j}$ satisfy the Vandermonde system, which well corresponds with the approximation of the fluxes $f\left(x_{i+1 / 2}\right)$ obtained in the finite volumes method.

Remark 3.3. A similar correspondence can be established for central differences (CD) schemes. In particular the analogs of CD2, CD4 and CD6 in Section 1 are PPM0, PPM1 and PPM2 of Section 2.

Remark 3.4. For the semi-Lagrangian schemes, we can also use the upwind approximations in the reconstruction instead of the PPM ones:

$$
P_{i}\left(x_{i-1 / 2}+\alpha \Delta x\right)=\left(3 \alpha^{2}-4 \alpha+1\right) f_{(i-1 / 2)^{+}}^{n}+\left(3 \alpha^{2}-2 \alpha\right) f_{(i+1 / 2)^{-}}^{n}+\left(6 \alpha-6 \alpha^{2}\right) \bar{f}_{i}^{n} \quad \text { with } \alpha \in[0,1]
$$

and $f_{(i+1 / 2)^{+}}^{n}\left(\operatorname{resp} . f_{(i+1 / 2)^{-}}^{n}\right)$ is reconstructed using $(4)$ with $s=d+1, r=-d+1($ resp. $s=-r=d)$. In the case $d=0,1$, this scheme coincides with LAG- $2 d+1$. For $d$ greater, it does not coincide with LAG- $2 d+1$ (since the reconstruction is still of degree three so that it has not the same degree as LAG-2d+1). but the "exponential integrator" limit (as defined in Proposition 3.2) will. In particular, we can gain a higher order of accuracy at the limit (see also [8]).

Remark 3.5. We can check that CD schemes preserve exactly the $L^{2}$ discrete norm $\sum_{j=0}^{N-1}\left|f_{j}(t)\right|^{2}$. On the other hand, upwind schemes make the $L^{2}$ norm decrease: we can check that

$$
\sum_{j=-d}^{d} a_{j}(\cos (j \omega)-\cos ((j-1) \omega)) \geq 0,0 \leq \omega \leq 2 \pi
$$

for $d=3$ for example, and this relation remains true from the stability of the LAG-2d +1 scheme, for all $d \in \mathbb{N}$. The conservation of the $L^{2}$ norm which is at first glance a good property is not so satisfactory, since it can generally lead to spurious oscillations. On the contrary, little dissipation, obtained with high order upwind approximation of the derivatives seems better in the limit regime. See also [14], for a discussion about odd and even order interpolation schemes. Note that this is a key point in [4]; there a non linear scheme is derived: central approximation is used where the solution is smooth and upwind approximation of one degree less is used where the solution is not smooth. We can also remark, that when not so small time steps are used, the $L^{2}$ norm generally decreases in a semi-Lagrangian scheme with centered reconstruction of the derivatives (e.g. cubic splines, PPM) and it can prevent from the spurious oscillations, that are observed in the finite volumes case. 
Remark 3.6. One could wonder about the existence of a time discretization of the finite volumes scheme so that it coincides with the semi-Lagrangian scheme, at least for $|a| \Delta t \leq \Delta x$. This can be achieved with a Cauchy-Kovalevsky procedure [23], as noticed in [14].

Remark 3.7. We have not specified how to compute the initial data $\bar{f}_{j}^{0}, j=0, \ldots, N-1$. Since we deal with finite volumes, a natural choice would be to use

$$
\bar{f}_{j}^{0}=\frac{1}{\Delta x} \int_{x_{j-1 / 2}}^{x_{j+1 / 2}} f(0, x) d x
$$

However, with that choice we loose the high order approximation. We will use instead the midpoint approximation

$$
\bar{f}_{j}^{0}=f\left(0, x_{j}\right)
$$

which leads to high order accuracy (sic!), since, in the semi-Lagrangian context, the scheme is then equivalent to the pointwise semi-Lagrangian scheme, as noticed in [13]. In [21], the authors classify this type of method in the finite difference semi-Lagrangian schemes (and not finite volumes) and present this type of scheme by introducing a function $h$ satisfying

$$
f\left(t_{n}, x_{j}\right) \simeq \frac{1}{\Delta x} \int_{x_{j-1 / 2}}^{x_{j+1 / 2}} h\left(t_{n}, x\right) d x
$$

which is then updated in a finite volumes manner. We emphasize that the equivalence only holds for the constant advection case. See [21] for details and other similar reconstructions, in the WENO framework.

\section{Numerical Results}

This section is devoted to the presentation of the numerical results of the different schemes described above. We focus on the Vlasov-Poisson test cases. Our results will be compared to reference semi-Lagrangian methods "Lag3" and "Lag5" (see [13,17] for more details).

For the VFSL2 method, we will consider two Gauss points in time and a Verlet algorithm for the research of the feet of the characteristics. The reconstruction is performed with a Lagrange 3 and 5 . Two versions are then considered, with or without splitting. These methods will be called Vfsl3 and Vfsl5 for the split approach and Vfsl3-ns, Vfsl5-ns for the unsplit approach. Some results will be also presented using PPM1 reconstruction with a splitting procedure.

For the finite volumes methods, we present the results for CD4 and UP5 together with a RK4 time integration. Note that in our test cases, the last term of Prop. 1.1 does not impact the numerical results.

Two test cases are studied, the Bump on tail (BOT) test presented in [24] and the two stream instability (TSI) (see [13]).

\subsection{Bump on tail}

The initial condition writes

$$
f_{0}(x, y)=\left(\frac{9}{10 \sqrt{2 \pi}} \exp \left(-\frac{y^{2}}{2}\right)+\frac{2}{10 \sqrt{2 \pi}} \exp \left(-2(y-4.5)^{2}\right)\right)(1+0.03 \cos (0.3 x)), \quad(x, y) \in[0, L] \times[-9,9]
$$

with $L=20 \pi$.

We consider the following numerical parameters: $N_{x}=N_{v}=128, \Delta t=0.01$. The Vlasov-Poisson model (1) preserves some physical quantities with time which will be used to compare the different methods. First, we 

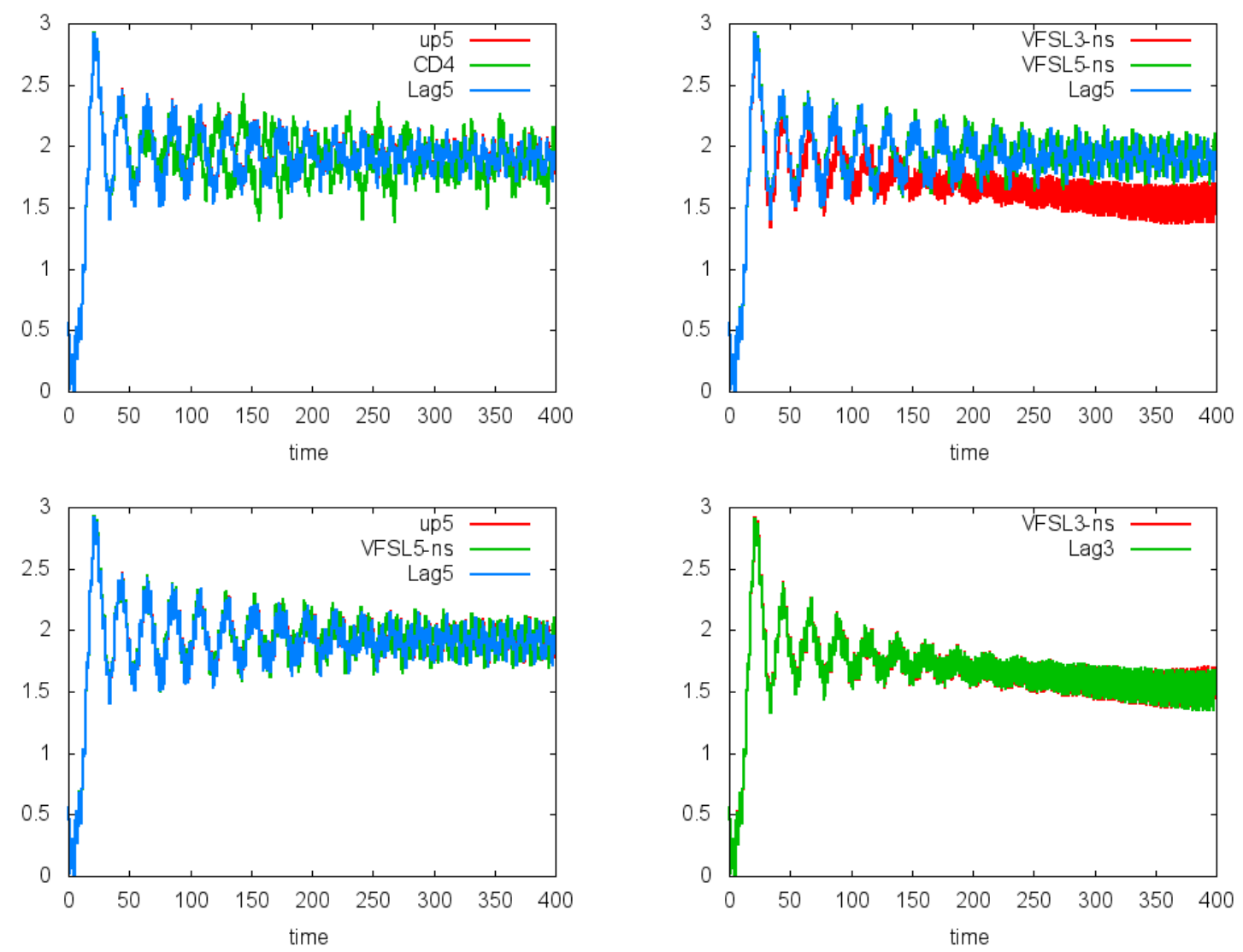

FiguRE 3. Bump on tail test: time evolution of the electric energy for "Banks" methods (CD4 and up5), for the unsplit Vfsl methods (Vfsl3-ns and Vfsl5-ns) and for semi-Lagrangian method (Lag5). $N_{x}=N_{v}=128, \Delta t=0.01$.

look at the time history of the $L^{p}$ norms of $f(p=1,2)$, but also the total energy $\mathcal{E}$ of the system, which is the sum of the kinetic energy $\mathcal{E}_{k}$ and the electric energy $\mathcal{E}_{e}$

$$
\mathcal{E}(t)=\mathcal{E}_{k}(t)+\mathcal{E}_{e}(t)=\int_{0}^{L} \int_{\mathbb{R}} f(t, x, y) \frac{y^{2}}{2} d y d x+\frac{1}{2} \int_{0}^{L} E^{2}(t, x) d x .
$$

On Figure 3, we plot the time evolution of the electric energy. First, we can observe the quite good behaviour of all the methods with regards to this diagnostic. The electric energy increases at the beginning (linear phase) and presents an oscillating behaviour for large times. It refers to a BGK type equilibrium composed of three vertices which are moving along the velocity of the initial bump on tail $v_{t}=4.5$. We also remark the fact that up5, Lag5 and Vfsl5 are very similar. Indeed Vfsl5 and Lag5 have the same reconstruction ; for up5 and Lag5, the link has been explained in Prop. 3.2. Obviously, the same is true for third order reconstruction methods Vfsl3-ns and Lag3.

Let us also remark the diffusive behaviour of the third order Lagrange based method (Vfsl3-ns and Lag3) which has been exhibited in [13] ; when fine structures are developed within the vertices, they are eliminated faster than when a higher order reconstruction is used (Lagrange 5 based methods like up5, Lag5, Vfsl5-ns). Hence the long time behaviour of the electric energy is better. 


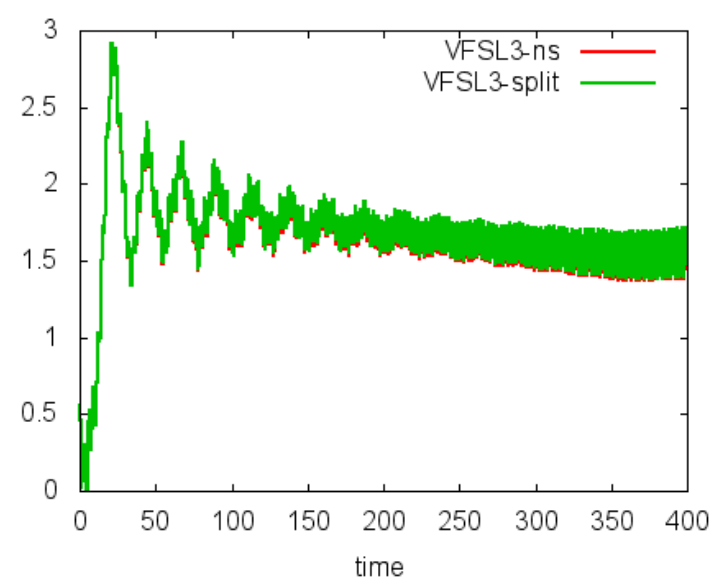

Figure 4. Bump on tail test: time evolution of the electric energy for Vfsl methods (unsplit Vfsl methods (Vfsl3-ns and Vfsl5-ns) and split ones for semi-Lagrangian method (Lag5). $N_{x}=$ $N_{v}=128, \Delta t=0.01$.

On Figure 4, we compare the split and unsplit version of Vfsl3. We can observe that the two versions are very similar which validates our approach. Indeed, in the Vlasov-Poisson context, the splitting procedure can be used and can be viewed as a reference solution. Similar figure is obtained for Vfsl5.

On Figure 5, we plot the time history of the total energy for the different methods. Except for the Lagrange 3 based method, we observe that this quantity is very well preserved. Note that this conservation is quite difficult to obtain and the use of high order reconstruction enables to get a good behaviour of the total energy.

On Figure 6, we are interested in the time history of the $L^{2}$ norm. We observed that CD4 preserves the $L^{2}$ norm very well whereas for the other ones, this quantity decreases in time. For the Lagrange 5 based methods, after the decrease around $t=50$ (which corresponds to a time of the creation of structures which are smaller than the size of the grid and then are eliminated by the scheme), we can observe that the $L^{2}$ norm is nearly constant, which is not the case of Lagrange 3 based methods. This also motivates the use of high order reconstructions.

On Figure 7, the evolution of the $L^{1}$ norm is plotted. What we see here is a contrary tendency of the previous diagnostic: CD4 presents a quite bad behaviour regarding the positivity compared to the other methods. Indeed, as mentioned in [4], CD4 presents oscillations that can not be viewed through the $L^{2}$ norm diagnostic, but which are emphasized on the $L^{1}$ norm diagnostic. We can also observe the results of up5, Vfsl5-ns and Lag5 methods which are very close.

\subsection{Two stream instability}

The initial condition is given by

$$
f_{0}(x, y)=\frac{1}{\sqrt{2 \pi}} \exp \left(-\frac{y^{2}}{2}\right)(1+0.05 \cos (0.5 x)), \quad(x, y) \in[0, L] \times[-9,9]
$$

with $L=4 \pi$.

We consider the following numerical parameters: $N_{x}=N_{v}=128$ and $\Delta t=0.005$. We present here 2D diagnostics of the full distribution function.

The results proposed in Figure 8 confirm the observations of the previous test case. Indeed, CD4 presents a lot of oscillations which lead to a poor quality. Moreover, the use of a third reconstruction (as for Vfsl3-ns) leads to a very smooth solution; when it is compared to a reference solution (plotted on Figure 9), we can 

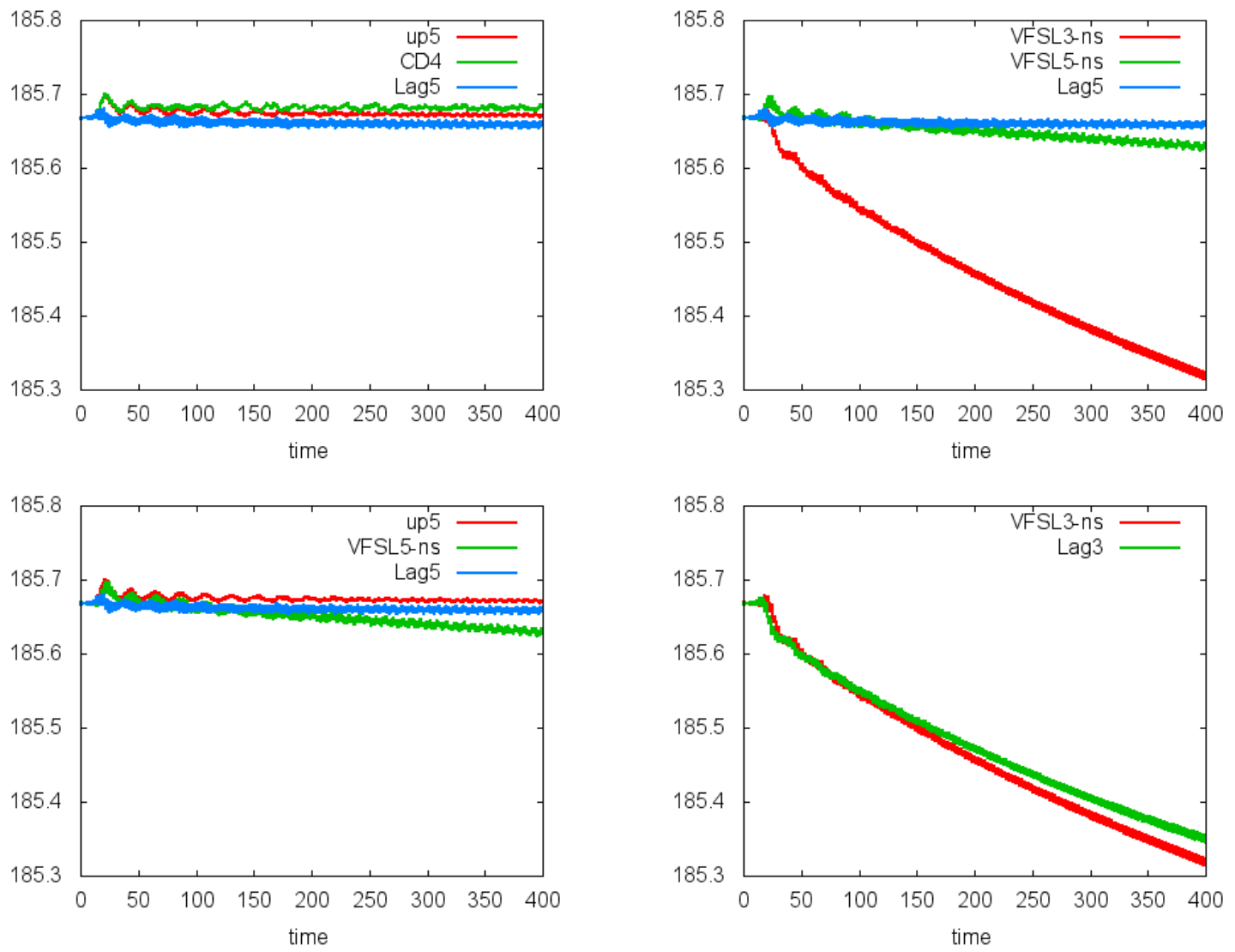

Figure 5. Bump on tail test: time evolution of the total energy for "Banks" methods (CD4 and up5), for the unsplit Vfsl methods (Vfsl3-ns and Vfsl5-ns) and for semi-Lagrangian method (Lag3 and Lag5). $N_{x}=N_{v}=128, \Delta t=0.01$.

see that the details have been eliminated by the scheme. When a higher order is used (as for up5 or Vfsl5ns), additional small structures are described. On Figures 10, 11, we again see the link between LAG3/up3, LAG5/up5 and also CD4/PPM1 for small $\Delta t$ as depicted in Remark 3.3. In particular, the bad oscillations of the centered reconstruction PPM1 are emphasized, when (very) small time steps are used, whereas the uncentered reconstructions LAG3 and LAG5 are insensitive to the decrease of time step. Note also that the PPM1 reconstruction behaves well when the time step is not too small, which is possible for a semi-Lagrangian scheme.

\section{Conclusion}

In this work, finite volumes schemes have been studied and compared for the numerical approximation of the Vlasov-Poisson system. The main goal was to develop unsplit methods for the Vlasov equation. Two kinds of methods have been discussed: ( $i$ ) a finite volume method inspired by [4], and (ii) a method based on Gauss points in time for the evaluation of the fluxes. These two methods have a good behaviour in the context of academic plasma test cases, compared to standard semi-Lagrangian methods. Moreover, a link have been performed between finite volumes methods and semi-Lagrangian methods for advection equations. In particular, when the time step $\Delta t$ tends to zero, semi-Lagrangian methods recovered some finite volumes method features. 

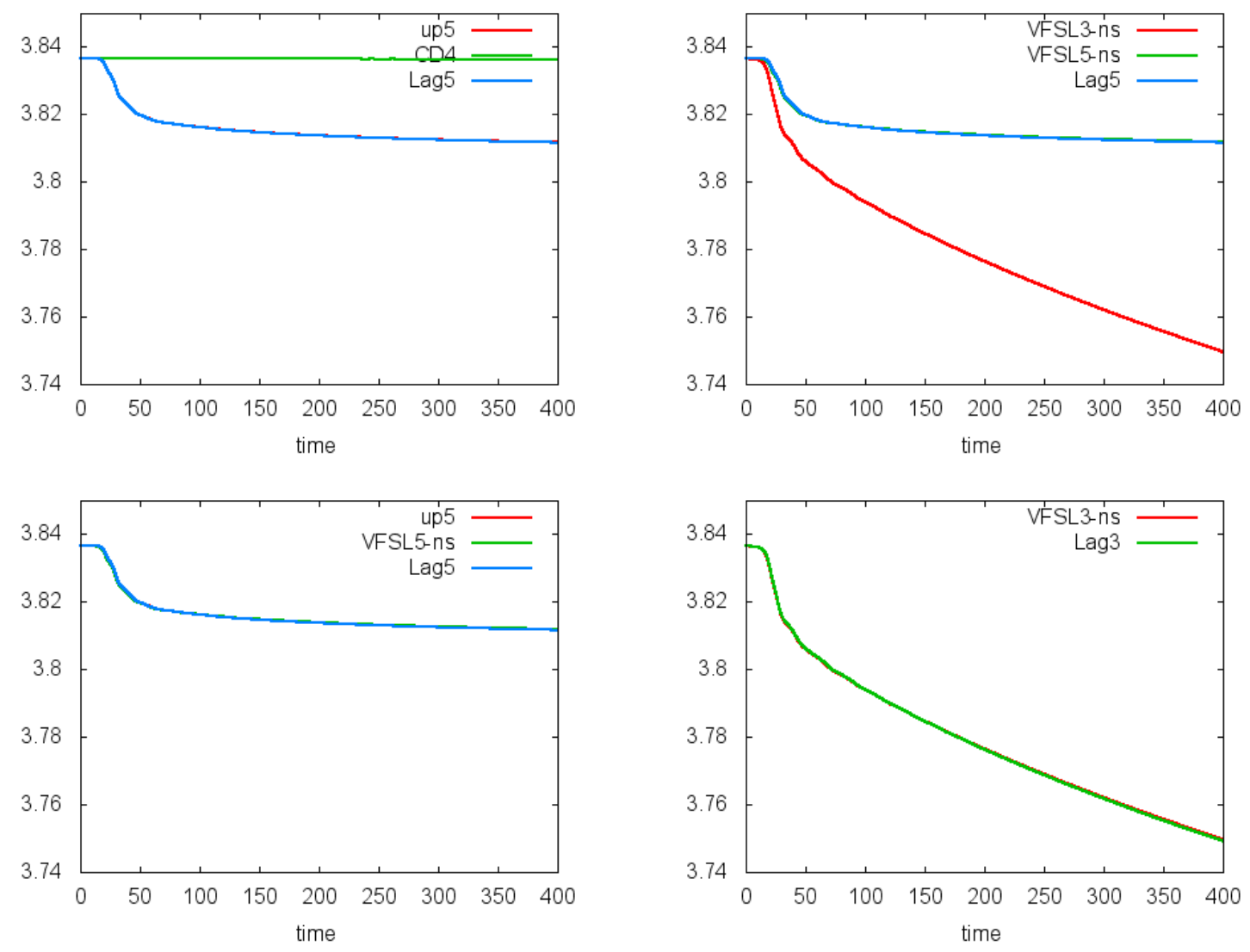

Figure 6. Bump on tail test: time evolution of the $L^{2}$ norm for "Banks" methods (CD4 and up5), for the unsplit Vfsl methods (Vfsl3-ns and Vfsl5-ns) and for semi-Lagrangian method (Lag3 and Lag5). $N_{x}=N_{v}=128, \Delta t=0.01$.

\section{REFERENCES}

[1] T. D. Arber, R. G. Vann, A critical comparison of Eulerian-grid-based Vlasov solvers, J. Comput. Phys. 180 (2002), pp. 339-357.

[2] M. BALDAuF, Stability analysis for linear discretisations of the advection equation with Runge-Kutta time integration, J. Comput. Phys. 227 (2008), pp. 6638-6659.

[3] C. K. Birdsall, A. B. Langdon, Plasma Physics via Computer Simulation, Inst. if Phys. Publishing, Bristol/Philadelphia, 1991.

[4] J. W. Banks, J. A. F. Hittinger, A new class of nonlinear finite-volume methods for Vlasov simulation, IEEE Trans. Plasma Sc 38, (2010).

[5] A. S. Bonnet-BenDhia, S. Fliss, P. Joly, P. Moireau, Introduction aux équations aux dérivées partielles et à leur approximation numérique, polycopié, cours ENSTA, 2011.

[6] J. P. Boris, D. L. Book, Flux-corrected transport. I: SHASTA, a fluid transport algorithm that works, J. Comput. Phys. 11 (1973), pp. 38-69.

[7] J. P. Braeunig, N. Crouseilles, V. Grandgirard, G. Latu, M. Mehrenberger, E. SonnendrüCker, Some numerical aspects of the conservative PSM scheme in a $4 D$ drift kinetic code, INRIA Report number 7109.

[8] F. Charles, B. Després, M. Mehrenberger, Enhanced convergence estimates for semi-Lagrangian schemes Application to the Vlasov-Poisson equation, INRIA Report number 00629081.

[9] C. Z. Cheng, G. Knorr, The integration of the Vlasov equation in configuration space, J. Comput. Phys. 22 (1976), pp. $330-3351$. 

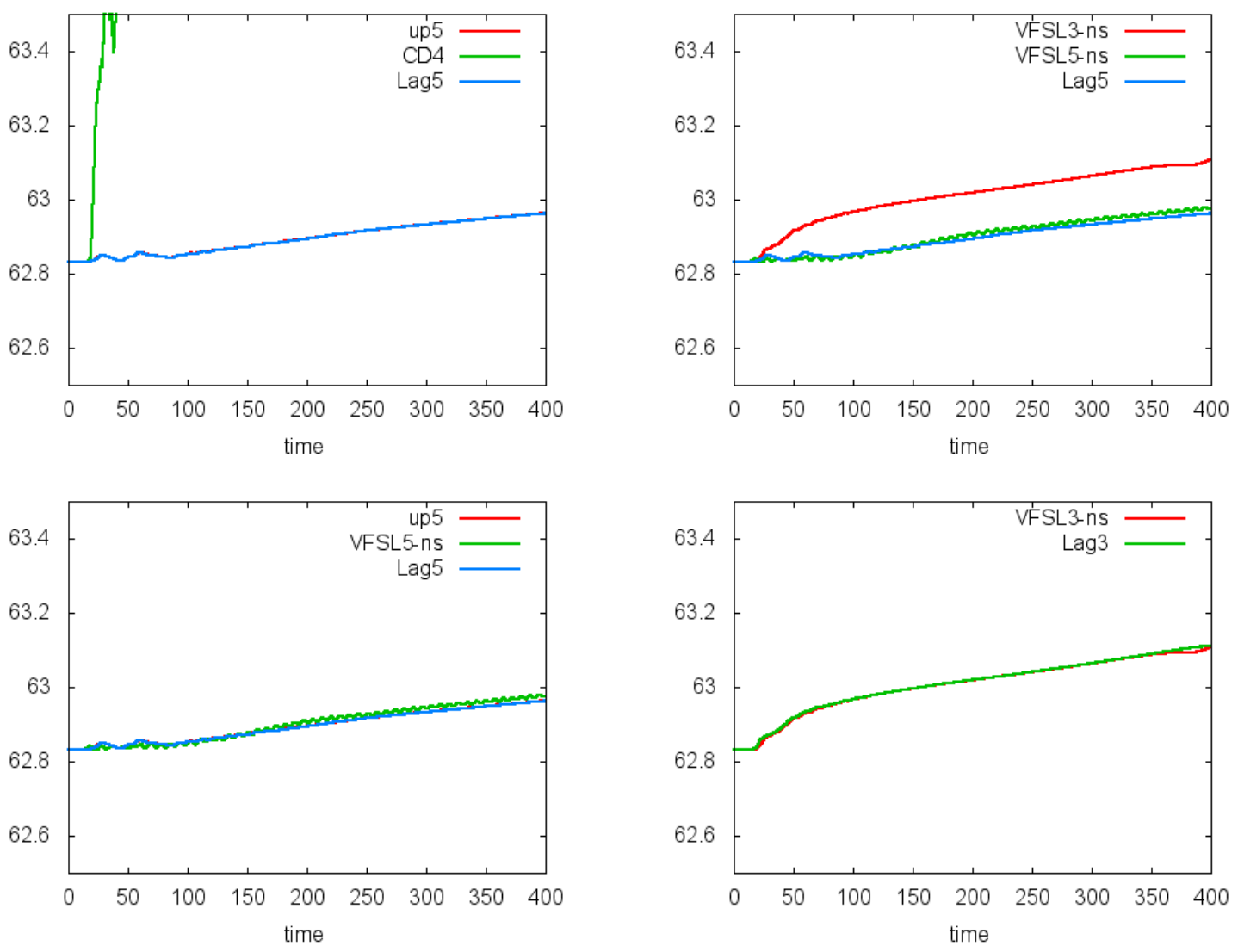

Figure 7. Bump on tail test: time evolution of the $L^{1}$ norm for "Banks" methods (CD4 and up5), for the unsplit Vfsl methods (Vfsl3-ns and Vfsl5-ns) and for semi-Lagrangian method (Lag3 and Lag5). $N_{x}=N_{v}=128, \Delta t=0.01$.

[10] P. Colella, P. R. Woodward, The piecewise parabolic method (PPM) for gas-dynamical simulations, J. Comput. Phys. 54 (1984), pp. 174-201.

[11] P. Colella, M. R. Dorr, J. A. F. Hittinger, D. F. Martin, High-Order, Finite-Volume Methods in Mapped Coordinates, [12] J. Comput. Phys. 230 (2011), pp. 2952-2976.

[13] N. Crouseilles, M. Mehrenberger, E. SonnendrüCker, Conservative semi-Lagrangian schemes for Vlasov equations, J. Comput. Phys. 229 (2010), pp. 1927-1953.

[14] B. DesPRÉs, Uniform asymptotic stability of Strang's explicit compact schemes for linear advection, SIAM J. Numer. Anal. 47 (2009), pp. 3956-3976.

[15] N. Elkina, J. Büchner, A new conservative unsplit method for the solution of the Vlasov equation, J. Comput. Phys. 213 (2006), pp. 862-875.

[16] E. Fijalkow, A numerical solution to the Vlasov equation, Comput. Phys. Commun. 116 (1999), pp. 329-335.

[17] F. Filbet, E. Sonnendrücker, P. Bertrand, Conservative numerical schemes for the Vlasov equation, J. Comput. Phys. 172 (2001), pp. 166-187.

[18] F. Filbet, E. Sonnendrücker, Comparison of Eulerian Vlasov solvers, Comput. Phys. Comm. 151 (2003), pp. $247-266$.

[19] F. Huot, A. Ghizzo, P. Bertrand, E. Sonnendrücker, O. Coulaud, Instability of the time splitting scheme for the one-dimensional and relativistic Vlasov-Maxwell system, J. Comput. Phys., 185 (2003), pp. 512-531.

[20] P.H. Lauritzen, R. D. Nair, P. A. Ullrich, A conservative semi-Lagrangian multi-tracer transport scheme (CSLAM) on the cubed-sphere grid, J. Comput. Phys. 229 (2010), pp. 1401-1424. 


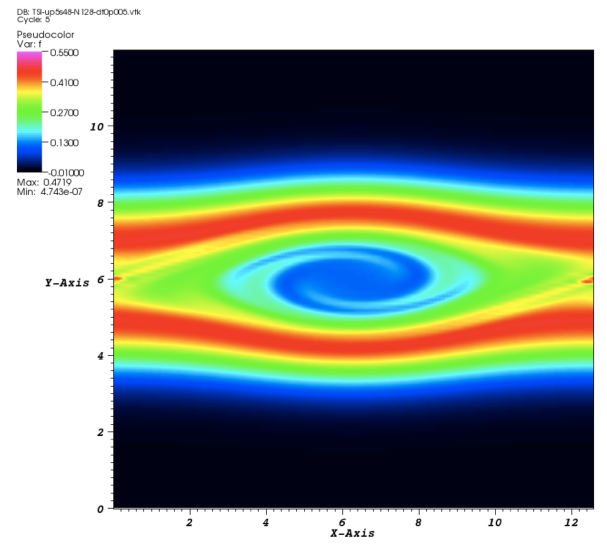

User: Michemenhrenberger
FriDec 909:38:09 2011

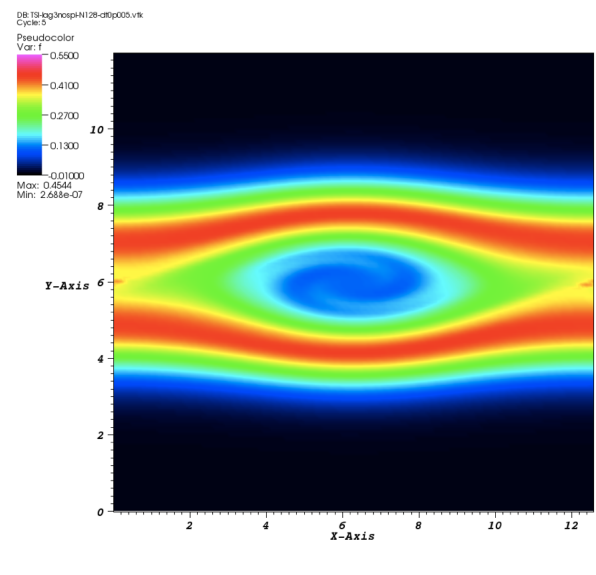

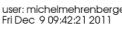

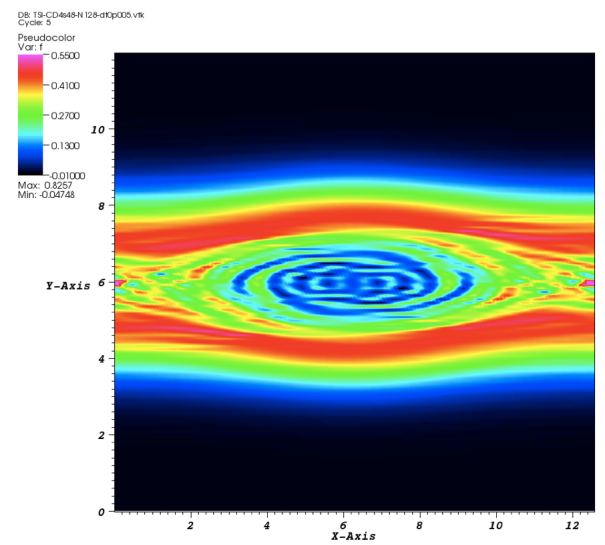

User: michememehrenberge
Fin Dec 909:42:28 2011

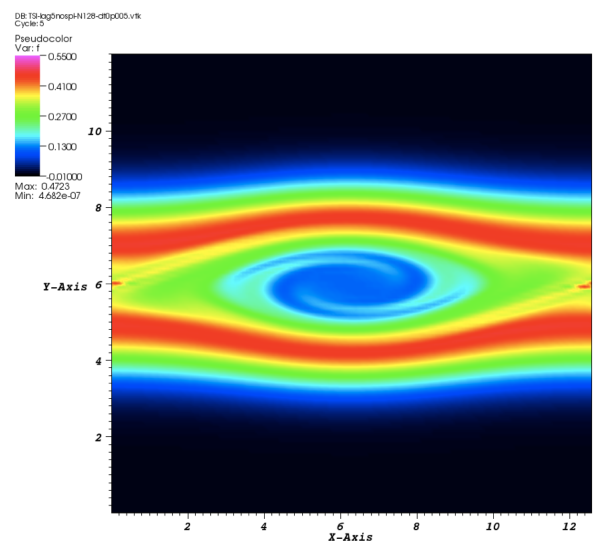

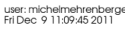

Figure 8. Two stream instability test: distribution function as a function of $x$ and $v$ at time $t=53$ for (from top to bottom and from left to right): up5, CD4, Vfsl3-ns, Vfsl5-ns. $N_{x}=N_{v}=128, \Delta t=0.005$.

[21] J.M. QIU, C. W. SHu, Conservative semi-Lagrangian finite difference WENO formulations with applications to the Vlasov equation, Comm. Comput. Phys. 10 (2011), pp.979-1000.

[22] J.M. QIU, C. W. SHu, Positivity preserving semi-Lagrangian discontinuous Galerkin formulation: theoretical analysis and application to the Vlasov-Poisson system, J. Comput. Phys., Volume 230, Issue 23 (2011), pp. 8386-8409.

[23] T. Schwartzkopff, M. Dumbser, C.D. Munz, Fast high order ADER schemes for linear hyperbolic equations and their numerical dispersion and dissipation, J. Comput. Phys. 197 (2004), pp. 532-538.

[24] M. SHOuCRI, Nonlinear evolution of the bump-on-tail instability, Phys. Fluids 22 (1979), pp. 2038-2039. 

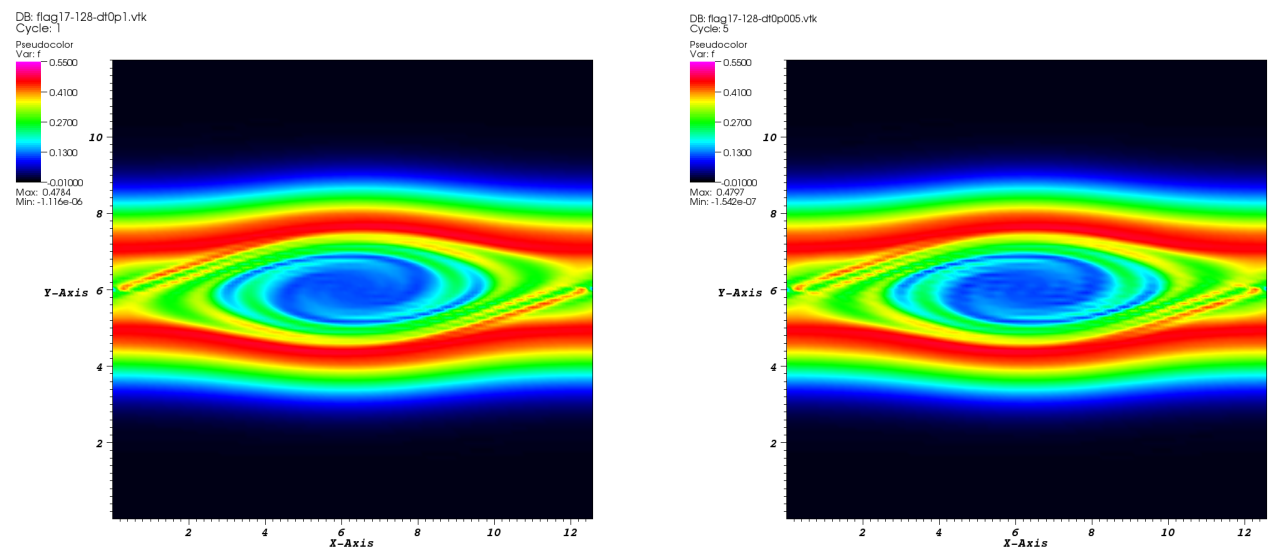

User: Micheimenrenberge
Mon Doc 12 14:28:08 2011

User: Michelmentrenberge
Mon Doc 12 14:31:32 2011
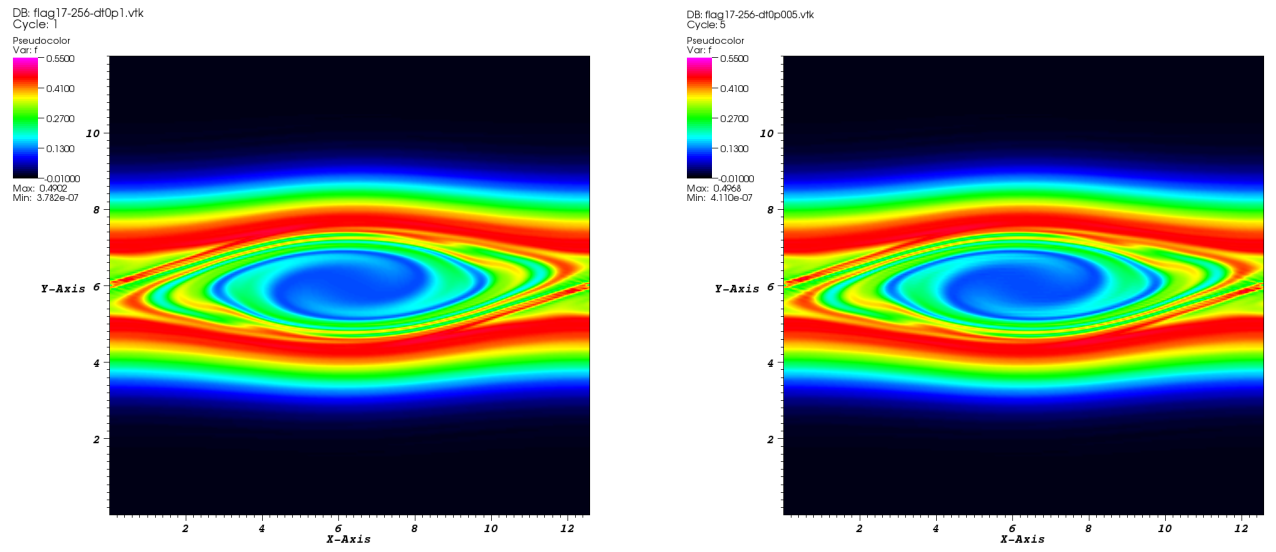

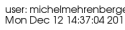

Figure 9. Two stream instability test: distribution function as a function of $x$ and $v$ at time $t=53$ for a semi-Lagrangian method with a Lagrange reconstruction of order 17 with $\Delta t=0.1$ (left), $\Delta t=0.005$ (right), and $N_{x}=N_{v}=128$ (top), $N_{x}=N_{v}=256$ (bottom). 

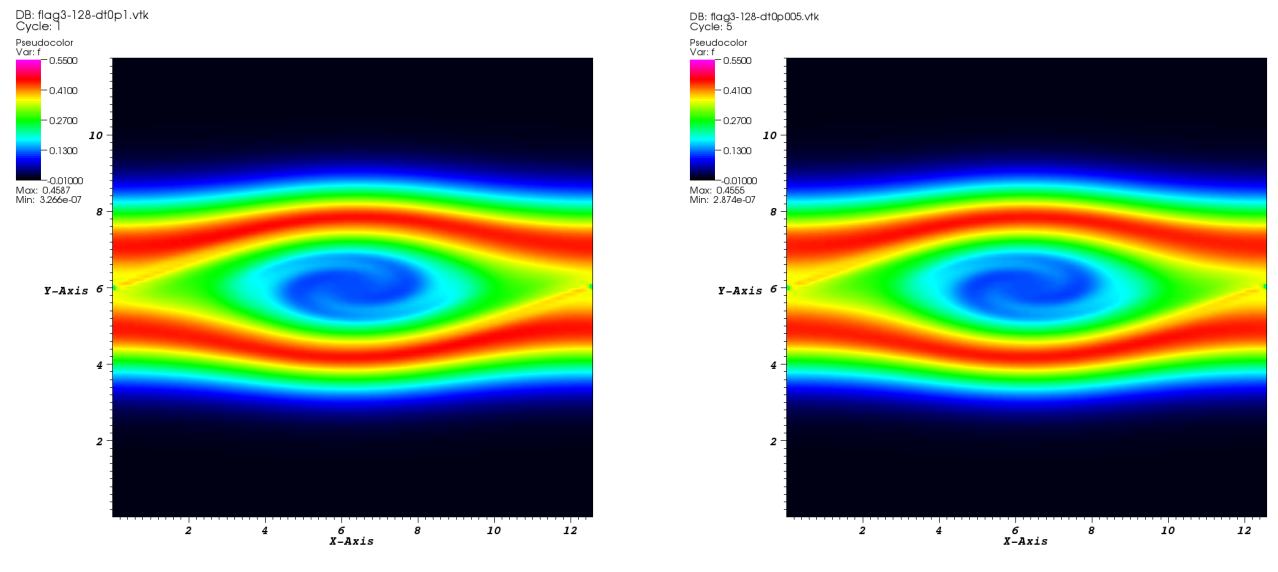

User: Michementrenberge
Tue Dec 1305:19:35 2011

User: Micheimenrenberge
Tue Doc $1305: 21: 242011$
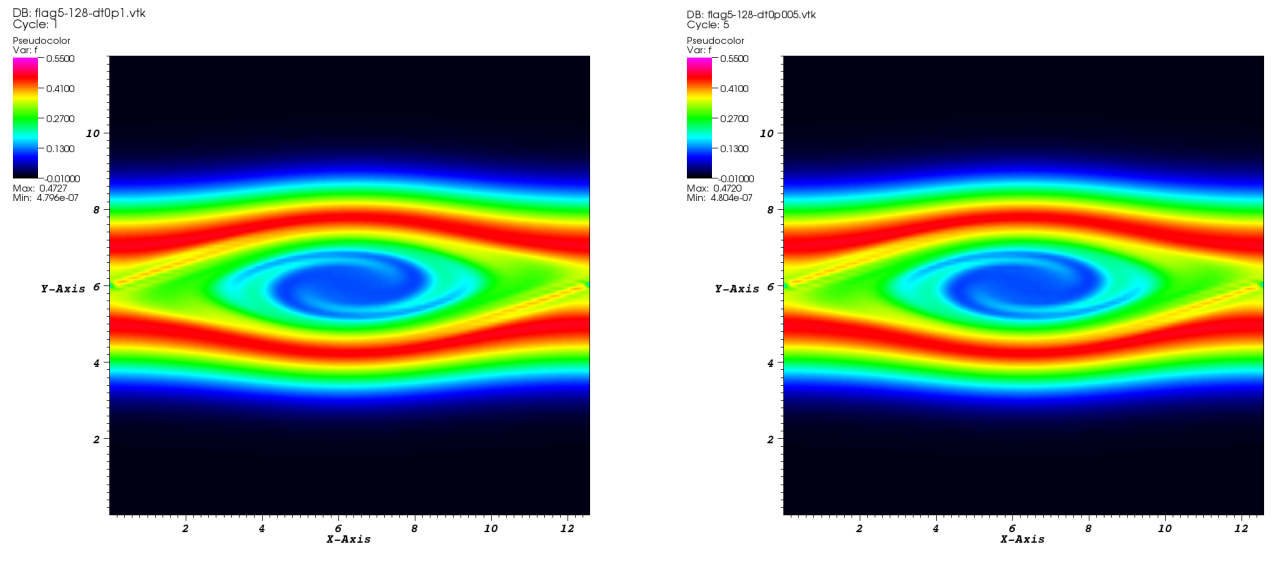

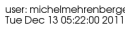

Figure 10. Two stream instability test: distribution function as a function of $x$ and $v$ at time $t=53$ for a semi-Lagrangian method with $N_{x}=N_{v}=128$ and a Lagrange reconstruction of order 3 (top), 5 (bottom) with $\Delta t=0.1$ (left), $\Delta t=0.005$ (right). 


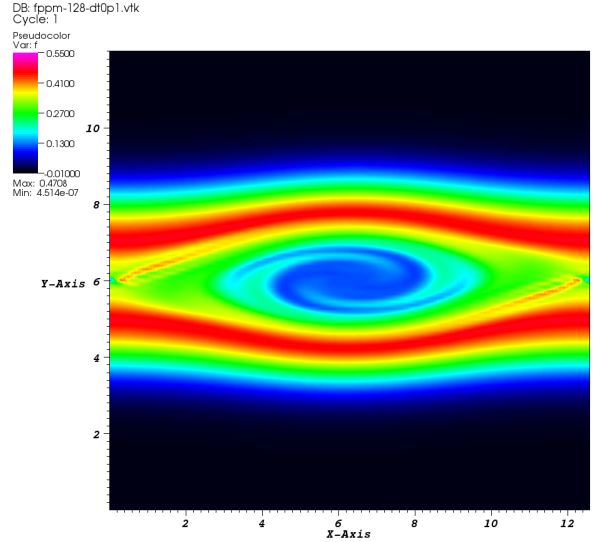

User: michementrenberger
Tue Doc $1305: 32: 1420111$

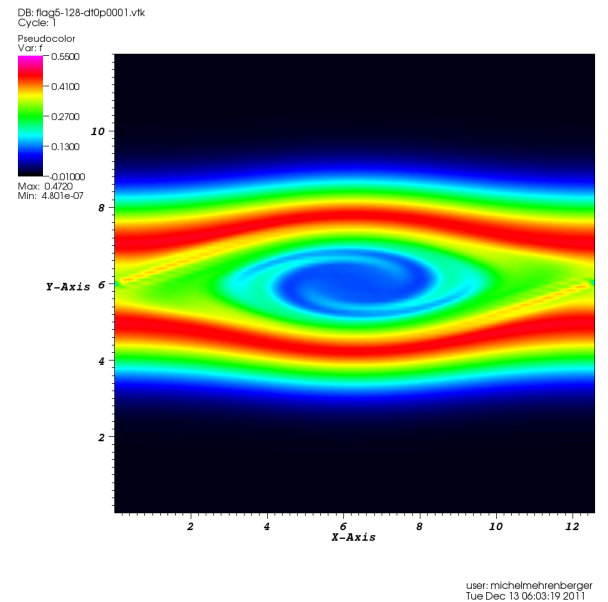

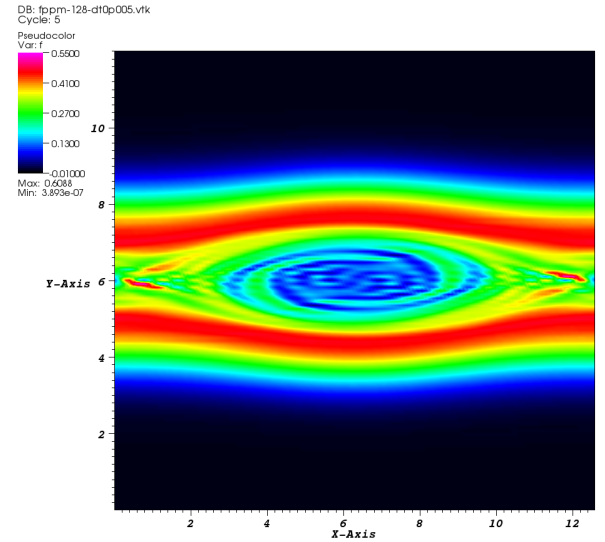

User: Michelmenrenberge
Tue Doc 1305 05:33:41 2011

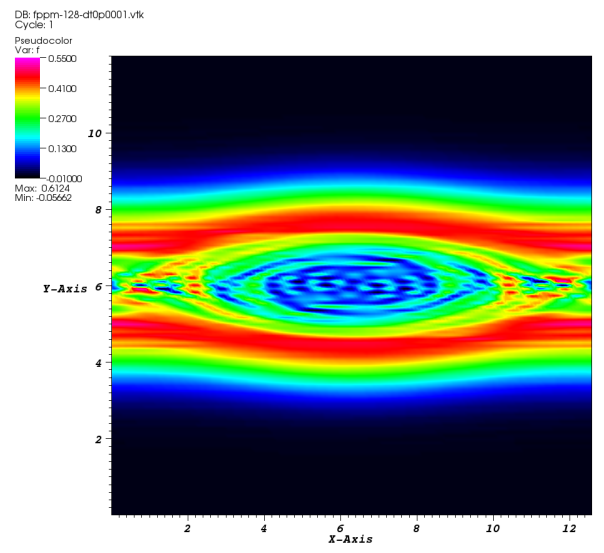

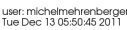

Figure 11. Two stream instability test: distribution function as a function of $x$ and $v$ at time $t=53$ for a semi-Lagrangian method with $N_{x}=N_{v}=128$ and PPM1 reconstruction with $\Delta t=0.1$ (top-left), $\Delta t=0.005$ (top-right), $\Delta t=0.0001$ (bottom-right), and Lagrange reconstruction of order 5 with $\Delta t=0.0001$ (bottom-left). 